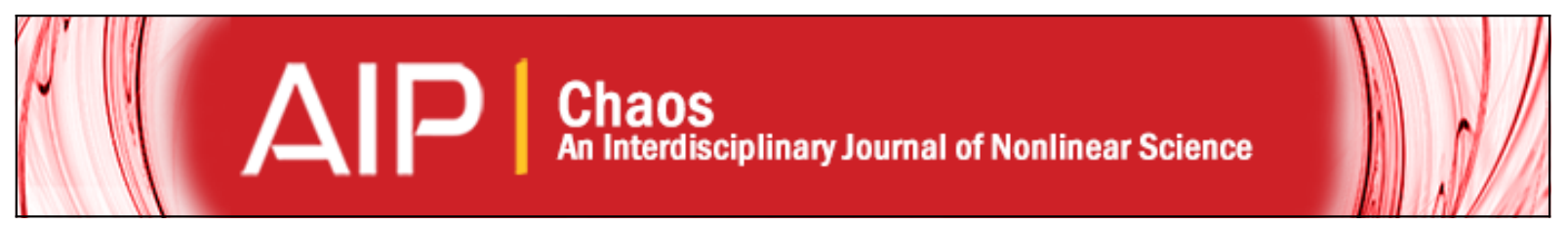

Lagrangian coherent structures are associated with fluctuations in airborne microbial populations

P. Tallapragada, S. D. Ross, and D. G. Schmale III

Citation: Chaos: An Interdisciplinary Journal of Nonlinear Science 21, 033122 (2011); doi: 10.1063/1.3624930

View online: http://dx.doi.org/10.1063/1.3624930

View Table of Contents: http://scitation.aip.org/content/aip/journal/chaos/21/3?ver=pdfcov

Published by the AIP Publishing

Copyright by the American Institute of Physics. Lagrangian coherent structures are associated with fluctuations in airborne microbial populations. Tallapragada, P. and Ross, S. D. and Schmale, D. G., Chaos: An

Interdisciplinary Journal of Nonlinear Science, 21, 033122 (2011), DOI:http:// dx.doi.org/10.1063/1.3624930

AIP Re-register for Table of Content Alerts 


\title{
Lagrangian coherent structures are associated with fluctuations in airborne microbial populations
}

\author{
P. Tallapragada, ${ }^{1}$ S. D. Ross, ${ }^{2}$ and D. G. Schmale $I I^{3}$ \\ ${ }^{1}$ Mechanical Engineering and Engineering Science, University of North Carolina at Charlotte, \\ North Carolina 28223, USA \\ ${ }^{2}$ Engineering Science and Mechanics, Virginia Tech, Blacksburg, Virginia 24061, USA \\ ${ }^{3}$ Plant Pathology, Physiology, and Weed Science, Virginia Tech, Blacksburg, Virginia 24061-0390, USA
}

(Received 27 April 2011; accepted 25 July 2011; published online 9 September 2011)

\begin{abstract}
Many microorganisms are advected in the lower atmosphere from one habitat to another with scales of motion being hundreds to thousands of kilometers. The concentration of these microbes in the lower atmosphere at a single geographic location can show rapid temporal changes. We used autonomous unmanned aerial vehicles equipped with microbe-sampling devices to collect fungi in the genus Fusarium $100 \mathrm{~m}$ above ground level at a single sampling location in Blacksburg, Virginia, USA. Some Fusarium species are important plant and animal pathogens, others saprophytes, and still others are producers of dangerous toxins. We correlated punctuated changes in the concentration of Fusarium to the movement of atmospheric transport barriers identified as finite-time Lyapunov exponent-based Lagrangian coherent structures (LCSs). An analysis of the finite-time Lyapunov exponent field for periods surrounding 73 individual flight collections of Fusarium showed a relationship between punctuated changes in concentrations of Fusarium and the passage times of LCSs, particularly repelling LCSs. This work has implications for understanding the atmospheric transport of invasive microbial species into previously unexposed regions and may contribute to information systems for pest management and disease control in the future. (C) 2011 American Institute of Physics. [doi:10.1063/1.3624930]
\end{abstract}

We consider the nonlinear dynamics underlying fluctuations of airborne microbe populations, via hypothesis testing combining theoretical considerations of atmospheric dynamical structures with atmospheric sampling and microbiological analysis. Our goal is to provide a new language for discussion of transport and mixing of atmospheric pathogens, paving the way for new modeling and management strategies for the spread of infectious diseases affecting plants, domestic animals, and humans. The hypotheses considered are built on the observation that in environmental flows, chaotic dynamical structure makes efficient movement and dispersal of agents possible, whether these agents are biological, chemical, or engineered devices like sensor platforms or delivery vehicles. Despite the flow complexity, our results suggest that atmospheric Lagrangian coherent structures play a significant role in geometrically organizing the motion of long-range microbe transport.

\section{INTRODUCTION}

Many spores, seeds, dust particles, and chemical pollutants can be transported across very large distances in the troposphere, sometimes on a continental scale. The atmosphere serves as a medium of transport as well as a reservoir for many of these particles. Several studies show the existence of such long range transport of dust, trace chemicals, and biota in the atmosphere. ${ }^{1-7}$ The dynamical systems approach to studying transport in geophysical flows has been around at least since the work of Pierrehumbert ${ }^{8,9}$ and has produced fruitful results. ${ }^{10-17}$ In this paper, we provide a description of sudden temporal changes in the concentration of fungi in the genus Fusarium collected with autonomous unmanned aerial vehicles (UAVs) at a single geographic location. In this paper, we explore the possibility that these "punctuated changes" in concentrations of Fusarium are related to the passage of large-scale, moving, transport barriers over the sampling location.

Many Fusarium species use the atmosphere to travel from one habitat to another, yet their atmospheric transport is poorly understood. Some members of the genus are important plant and animal pathogens, others saprophytes, and still others are producers of dangerous toxins. ${ }^{18-20}$ Atmospheric transport of Fusarium can be broadly categorized into three distinct stages ${ }^{7-14}$-(1) release and ascent of Fusarium into the planetary boundary layer (PBL), (2) long range transport of Fusarium in the PBL or above, and (3) deposition of Fusarium into a new habitat. Fig. 1 illustrates these three stages schematically.

In the release and ascent stage, spores of Fusarium (or any passively moving particles) have to cross a relatively thin boundary layer that can extend in height up to $50 \mathrm{~m}$, called the surface boundary layer (SBL). The SBL is the lowest "layer" of the atmosphere and, being in contact with the ground, it has very strong vertical gradients in wind speed, temperature, and humidity. The flow in this layer is highly turbulent because of the surface effects. ${ }^{21,22}$ The motion of spores in this layer can be approximated as random and indeed the Fokker-Planck equation is one way to 
Free atmosphere

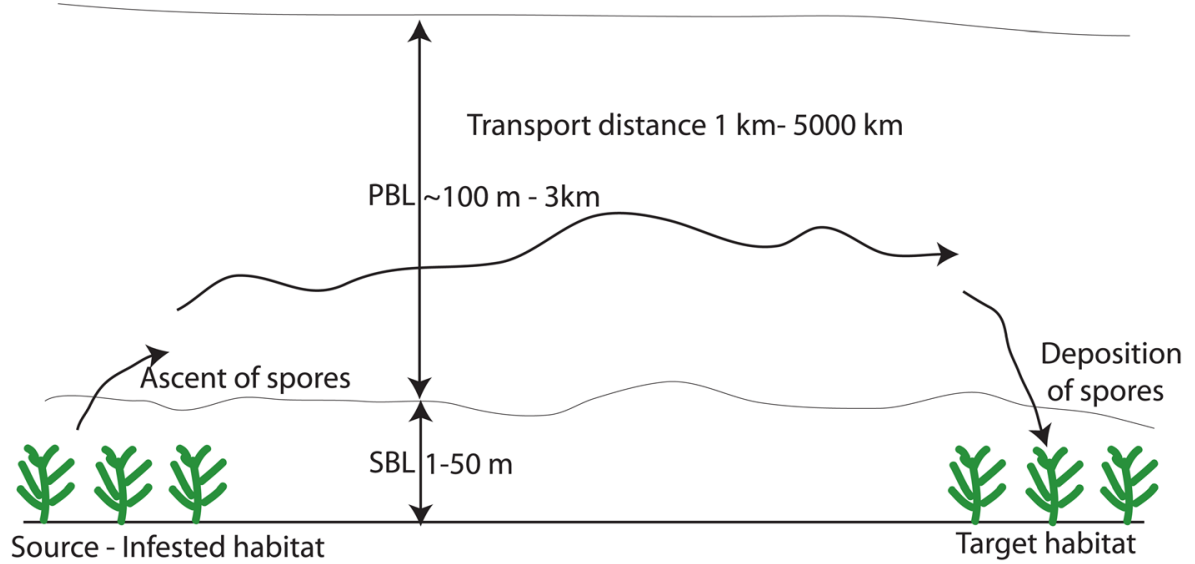

FIG. 1. (Color online) The three stages in the long range transport of microbes in the genus Fusarium.

describe their motion in this layer. ${ }^{23}$ Above the SBL is the PBL, a well-mixed layer in which the turbulence due to the surface forcing decreases. The height of the PBL extends from $\sim 50 \mathrm{~m}$ to $\sim 3 \mathrm{~km}$ above the ground, with the upper limit decreasing to as little as $300 \mathrm{~m}$ during the night. Once spores manage to cross the SBL and enter the PBL, they have the potential to be transported over long distances. Indeed, in the PBL and above, on the meso- to synoptic$(\sim 10-100 \mathrm{~km})$ scale, the motion over time-scales of a day is quasi-two-dimensional (2D) along constant pressure surfaces which parallel the landscape, following the homogeneous geostrophic equations appropriate to this scale. ${ }^{21,24}$

The first and third stages have received considerable attention in terms of developing computational models, for example, in the works of Aylor, Schmale, and co-workers. $^{23,25-28}$ The long range transport stage has usually been studied using a few sample trajectories. ${ }^{29-31}$ However, the study of individual trajectories cannot fully resolve the complex motion of the atmosphere, nor the resulting changes in the observed concentrations of Fusarium in the lower atmosphere. Moreover, studies using individual trajectories may rely on long integration time (days to weeks) for trajectories, which can lead to many uncertainties in trajectory computations. Rather than individual trajectories, the qualitative properties of sets of closely spaced trajectories are more reliable. Here, we propose a more systematic, geometric framework for discussing the changes in atmospherically advected microorganism concentrations, based on computations of atmospheric transport barriers (ATBs). The ATBs are approximated as Lagrangian coherent structures (LCSs) extracted as ridges from the finite-time Lyapunov exponent (FTLE) field. The LCSs serve as the most important material surfaces within the flow which effectively separate air masses of qualitatively different dynamics and, hence, are distinguished transport barriers. We hypothesized that punctuated changes in the concentration of Fusarium at a single geographic location are correlated with the passage of atmospheric LCSs. We test this hypothesis by integrating with experimental measurements of the concentration of dynamical systems framework for computing LCS during periods surrounding our Fusarium collections.

The paper is organized as follows. In Sec. II, we review the formulation of the FTLE and LCS. We also describe the so-called partial FTLE as the FTLE restricted to a lower dimensional manifold; in our case, a two-dimensional isobaric surface within the three-dimensional atmospheric flow. In Sec. III, we describe the method of collection of Fusarium spores in the atmosphere, the calculations for spore concentrations, and our definition for punctuated changes. We also present our hypotheses on the correlation of punctuated changes with the movement of LCSs and review the statistical method of contingency tables for hypothesis testing. In Sec. IV, we describe in detail the specific computational methods and simplifications we made for the computation of FTLE, the extraction of ridges from the FTLE field, and the identification of the passage of an LCS over the sampling point. In Sec. V, we provide detailed results of the movement of forward and backward LCS for sequential collections of Fusarium that show distinct changes in the concentrations of Fusarium. We also show the results of hypothesis testing for the other samples.

\section{MATHEMATICAL BACKGROUND ON LCS}

We begin with a review of the basic definitions and results pertaining to LCSs. Let $\phi: M \times \mathbb{R} \times \mathbb{R} \mapsto M$ be a smooth time-dependent flow map on a Euclidean manifold $M \subset \mathbb{R}^{\mathbb{1}}$ of dimension $n$, with the associated vector field $\dot{\mathbf{x}}=\mathbf{f}(\mathbf{x}, t)$. Given the context of atmospheric flow, we will be considering $n=2$ or 3 . The flow map $\phi$, considered point wise, maps a point $\mathbf{x}_{0} \in M$ at time $t_{0} \in \mathbb{R}$ to a point $\mathbf{x} \in M$ at time $t \in \mathbb{R}$, given by $\mathbf{x}\left(t ; t_{0}, \mathbf{x}_{0}\right)=\phi\left(\mathbf{x}_{0}, t_{0}, t\right)$. To simplify notation, we write $\phi\left(\mathbf{x}_{0}, t_{0}, t\right)$ as $\phi_{t_{0}}^{t}\left(\mathbf{x}_{0}\right)$, thus $\mathbf{x}_{0} \longmapsto \phi_{t_{0}}^{t}\left(\mathbf{x}_{0}\right)=\mathbf{x}$, where it is understood that $\phi_{t_{0}}^{t}: M \longmapsto M$ is a two-parameter family of maps, with parameters $t_{0}$ and $t$.

Consider a reference trajectory passing through the point $\mathrm{x}$ and a displaced, nearby trajectory passing through $\mathrm{x}+\delta \mathrm{x}$, both at time $t_{0}$. The flow map $\phi_{t_{0}}^{t}$ maps these points to $\phi_{t_{0}}^{t}(\mathbf{x})$ and $\phi_{t_{0}}^{t}(\mathbf{x}+\delta \mathbf{x})$ at time $t$. The displacement $\delta \mathrm{x}\left(t_{0}\right)$ evolves to $\delta \mathrm{x}(t)$ given by the Taylor expansion

$$
\delta \mathbf{x}(t)=\phi_{t_{0}}^{t}(\mathbf{x}+\delta \mathbf{x})-\phi_{t_{0}}^{t}(\mathbf{x})=\frac{d \phi_{t_{0}}^{t}}{d \mathbf{x}} \delta \mathbf{x}\left(t_{0}\right)+O\left(\left\|\delta \mathbf{x}^{2}\left(t_{0}\right)\right\|\right) .
$$

The norm or magnitude of $\delta \mathrm{x}\left(t_{0}\right)$ can be found using the standard inner product on $\mathbb{R}^{\mathrm{n}}$, 


$$
\begin{aligned}
\|\delta \mathbf{x}(t)\| & =\sqrt{\left\langle\frac{d \phi_{t_{0}}^{t}}{d \mathbf{x}} \delta \mathbf{x}\left(t_{0}\right), \frac{d \phi_{t_{0}}^{t}}{d \mathbf{x}} \delta \mathbf{x}\left(t_{0}\right)\right\rangle} \\
& =\sqrt{\left\langle\delta \mathbf{x}\left(t_{0}\right), \mathbf{C} \delta \mathbf{x}\left(t_{0}\right)\right\rangle}
\end{aligned}
$$

where $\mathbf{C}=\left(\frac{d \phi_{t_{0}}^{t}}{d \mathbf{x}}\right)^{*}\left(\frac{d \phi_{t_{0}}^{t}}{d \mathbf{x}}\right)$ is the finite-time right CauchyGreen deformation tensor, a rotation-independent measure of deformation, and ${ }^{*}$ denotes the transpose. The maximum growth of a displacement is given by the maximum principal stretch, i.e., by the maximum eigenvalue of $\mathrm{C}$,

$$
\max \|\delta \mathbf{x}(t)\|=\sqrt{\lambda_{\max }(\mathbf{C})}\left\|\delta \mathbf{x}\left(t_{0}\right)\right\| \xi_{1}\left(\mathbf{x}, t_{0}\right),
$$

where $\xi_{1}\left(\mathrm{x}, t_{0}\right)$ is the eigenvector of $C$ associated with $\lambda_{\max }$. The growth in the displacement depends on the initial point $\mathrm{x}$, initial time $t_{0}$, and the evolution or integration time $T=t-$ $t_{0}$. The maximum FTLE is defined as

$$
\sigma\left(\mathbf{x}, t_{0}, T\right)=\frac{1}{|T|} \log \left(\sqrt{\lambda_{\max }(\mathbf{C})}\right) .
$$

LCSs are defined to be ridges in the FTLE field. ${ }^{32,33}$ For $T>0$, these are the repelling LCSs which maximize the rate of time averaged stretching of trajectories around them. Attracting LCSs are defined as repelling LCSs in backward time.

We simplify the standard formulation of FTLE-LCS by using the concept of partial FTLE and partial LCS, as in Refs. 34-36. For example, for a time-dependent flow in $\mathbb{R}^{3}$, given by

$$
\begin{aligned}
& \left(x_{1}(t), x_{2}(t), x_{3}(t)\right) \\
& =\left(\phi_{1}\left(x_{1}, x_{2}, x_{3}, t_{0}, t\right), \phi_{2}\left(x_{1}, x_{2}, x_{3}, t_{0}, t\right), \phi_{3}\left(x_{1}, x_{2}, x_{3}, t_{0}, t\right)\right)
\end{aligned}
$$

one might be interested in the sensitivity of trajectories in the horizontal $x_{1}-x_{2}$ subspace with respect to initial perturbations in the $x_{1}-x_{2}$ subspace, then one need not consider the total deformation gradient,

$$
\left(\frac{d \phi}{d \mathbf{x}}\right)=\left(\begin{array}{lll}
\frac{\partial \phi_{1}}{\partial x_{1}} & \frac{\partial \phi_{1}}{\partial x_{2}} & \frac{\partial \phi_{1}}{\partial x_{3}} \\
\frac{\partial \phi_{2}}{\partial x_{1}} & \frac{\partial \phi_{2}}{\partial x_{2}} & \frac{\partial \phi_{2}}{\partial x_{3}} \\
\frac{\partial \phi_{3}}{\partial x_{1}} & \frac{\partial \phi_{3}}{\partial x_{2}} & \frac{\partial \phi_{3}}{\partial x_{3}}
\end{array}\right)
$$

since the appropriate partial deformation gradient,

$$
\left(\frac{d \phi}{d \mathbf{x}}\right)_{\text {partial }}=\left(\begin{array}{ll}
\frac{\partial \phi_{1}}{\partial x_{1}} & \frac{\partial \phi_{1}}{\partial x_{2}} \\
\frac{\partial \phi_{2}}{\partial x_{1}} & \frac{\partial \phi_{2}}{\partial x_{2}}
\end{array}\right)
$$

provides the needed information. This is particularly useful for the atmosphere, where the vertical wind velocity is at least 2 orders of magnitude smaller than the horizontal velocity. ${ }^{21,22,37}$ Furthermore, the trajectories of rising or falling air parcels are almost on isobaric surfaces on a 24-h time-scale in the PBL and for much longer in the free troposphere. ${ }^{21,22,37}$

By choosing pressure as the vertical coordinate, $x_{3}$, we can consider the trajectories to be restricted to a two-dimensional (isobaric) manifold on a 24-h time-scale, making the last row of the deformation gradient in Eq. (6) negligible. Pairs of air parcels that originate in the PBL on isobaric surfaces, which have only a small pressure difference between them, have a small difference in the horizontal velocities. This small difference in velocities on closely spaced isobaric surfaces can lead to horizontal shear stretching of vertical material lines. On a $24 \mathrm{~h}$ time scale, the vertical shear stretching of vertical material lines can be much higher than the horizontal stretching of horizontal line elements. This shear stretching is captured by the terms $\frac{\partial \phi_{1}}{\partial x_{3}}$ and $\frac{\partial \phi_{2}}{\partial x_{3}}$ in Eq. (6). We ignore these terms because they identify shear stretching. The intersection of the true LCSs computed from Eq. (6) with isobaric surfaces is approximately equal to the partial FTLE obtained from Eq. (7). Thus, the two-dimensional transport barriers can approximately be identified by piecing together the onedimensional transport barriers on isobaric surfaces, as illustrated in Fig. 2. Due to the shear between isobaric surfaces, the partial FTLE are not exactly vertically stacked on top of each other, but have slightly different position on different isobaric surfaces. In general, the partial FTLE field can be defined using any submatrix of the deformation gradient. However, the partial FTLE field will approximate the true FTLE only if the growth of perturbations in the ignored directions is negligible or converge to zero. Our interest, however, is not the specific value of the FTLE, but rather the topological features of the FTLE, e.g., the location of high ridges.

\section{ROLE OF ATBS IN PUNCTUATED CHANGES}

It is well-established that FTLE-LCS are time-dependent analogues of stable and unstable invariant manifolds of fixed point in time-dependent velocity fields and, more importantly, are almost material surfaces in geophysical

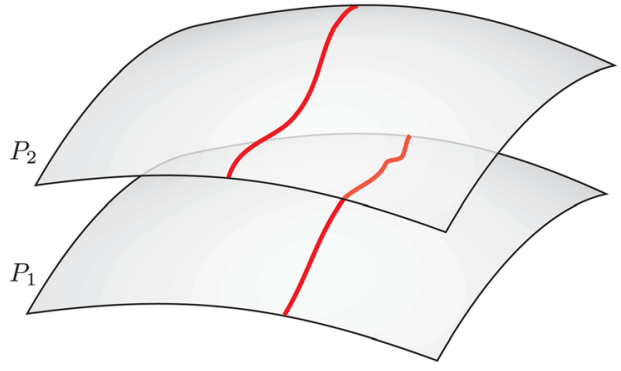

(a)

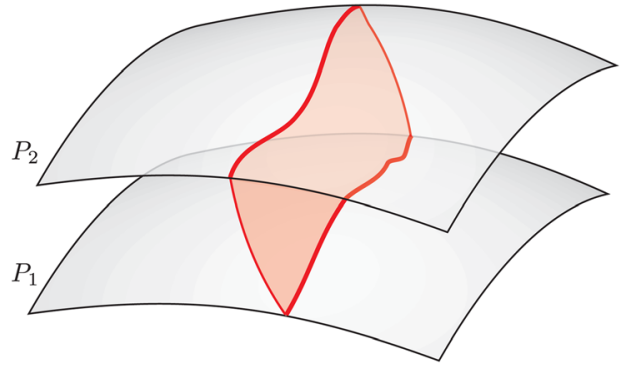

(b)
FIG. 2. (Color online) One-dimensional partial LCS, shown as curves on twodimensional isobaric surfaces with pressures $P_{1}$ and $P_{2}$, as in (a), can be pieced together to form the two-dimensional LCS in the full three-dimensional space, shown by the vertical surface, as in (b). 
flows of interest. ${ }^{12,15,32,33,36,38}$ These properties, along with their primary property of maximizing time-averaged repulsion over a finite-time horizon, ${ }^{39}$ effectively make them the most important barriers to the atmospheric transport of microorganisms such as Fusarium. The role of LCSs as transport barriers has been studied before in problems such as transport of pollutants in the ocean, ${ }^{12}$ the splitting of the ozone hole,${ }^{15}$ mixing and turbulence in jet streams, ${ }^{16}$ invariant tori-like LCSs in geophysical flows, ${ }^{40}$ locomotion and feeding mechanisms of jelly fish, ${ }^{41,42}$ segregation of inertial particles ${ }^{34}$ and the detection of low altitude shear inducing flow structures near airports. ${ }^{34}$ In particular, the role of transport barriers as separatrices that influence both mixing and separation processes has been investigated in many problems of geophysical flows. ${ }^{11,12,15}$ However, the relationship between the movement of atmospheric LCSs over the landscape and punctuated changes in concentration of microorganisms at a fixed geographic locations has not yet been investigated. In this section, we present hypotheses on the role of LCSs in punctuated changes in atmospheric concentrations of the fungal genus Fusarium.

\section{A. Punctuated changes in the concentration of Fusarium}

Microbes belonging to the genus Fusarium were selected for this study because of the relative abundance of Fusarium in the atmosphere ${ }^{43}$ and the existence of reliable methods for selectively collecting Fusarium in the lower atmosphere. ${ }^{44,45}$ Moreover, many members of this genus cause important diseases in animals and crops with serious economic consequences, as discussed in Sec. I.

Autonomous UAVs were used to collect colonies of $\mathrm{Fu}$ sarium from the lower atmosphere at Virginia Tech's Kentland Farm, $\left(37.1971^{\circ} \mathrm{N}\right.$ latitude and $80.5738^{\circ} \mathrm{W}$ longitude), from August 2006 to March 2010. These flights were conducted at a target altitude of $100 \mathrm{~m}$ above ground level, with a standard error of about $\pm 1 \mathrm{~m} .{ }^{44} \mathrm{~A}$ total of 100 flights were conducted, spread over all four seasons, with each flight lasting between 10 and $15 \mathrm{~min}$. The UAVs carried collection plates containing a Fusarium selective medium on the wings. ${ }^{46}$ The collection plates were incubated in the laboratory to allow colonies to develop. Fig. 3 shows one of the UAVs and collection plates after the colonies developed (i.e., viable spores that germinated and grew on the plates).
Let the number of spores sampled in a flight be denoted by $N_{p}$. Our autonomous UAV sampling scheme (consistent airspeed, sampling pattern, and altitude throughout the sampling missions) provides us with a robust measure of $N_{p}$ and, thus, of the calculated aerial concentration of Fusarium. Recently, Schmale designed a new aerobiological sampling device for autonomous UAVs with eight different petri plates (two plates on each of four arms; for comparison, the UAV in Fig. 3 has two on each arm). The servos that controlled the arms were synchronized to allow two arms with four inner plates to be opened, while the other two outer arms with four outer plates were closed. This allowed the UAV to collect two independent samples over the course of a single flight (the four inner plates were opened for $10 \mathrm{~min}$ and closed, and the four outer plates were opened for $10 \mathrm{~min}$ and closed). In 2010 and 2011, 19 such flights of dual-sampling were conducted. The colony counts $\left(N_{p}\right)$ between the consecutive $10 \mathrm{~min}$ flights were remarkably correlated, with a correlation coefficient of 0.93 $(p<0.001)$. Thus, the errors introduced by the sampling mechanism are very small. Analysis of the differences between the consecutive flights provides error bars on $N_{p}$ of $\pm 14 \%$. This result also suggests that the spatiotemporal gradient of the concentration is smooth (at least on the $\sim 20$ min timescale). Any observed dynamics in these concentrations over time can be attributed to factors other than the accuracy of the sampling regime, e.g., an association with atmospheric structures.

The number of colonies on the collection plates were counted $\left(N_{p}\right)$, and the concentration of Fusarium spores collected (number per unit volume) during the sampling event was estimated using the method given in Refs. 47 and 48 , which we review here. The UAVs used for collection of $F u$ sarium were flown in a circular flight path of radius approximately $100 \mathrm{~m}$ at a nearly constant speed of $60.0 \pm 1 \mathrm{~km} / \mathrm{h}$. Due to the circular path, the average relative velocity of the air with respect to the UAV is approximately the same as the speed of the UAV, as the upwind and downwind contributions of the wind speed cancel each other. Let the average air speed by $U$, the flight time by $T_{f}$, the radius of a single petri plate by $r$, the area of a single petri plate by $A_{e}=\pi r^{2}$, the number of Petri plates by $n$ and the effective volume of air sampled by the petri plates on the UAV by $V=n U T_{f} A_{e}$, and the aerial concentration of spores by $C$. To obtain the concentration $C$ from $N_{p}$ and $V$, we used approximations determined elsewhere, ${ }^{47,48}$

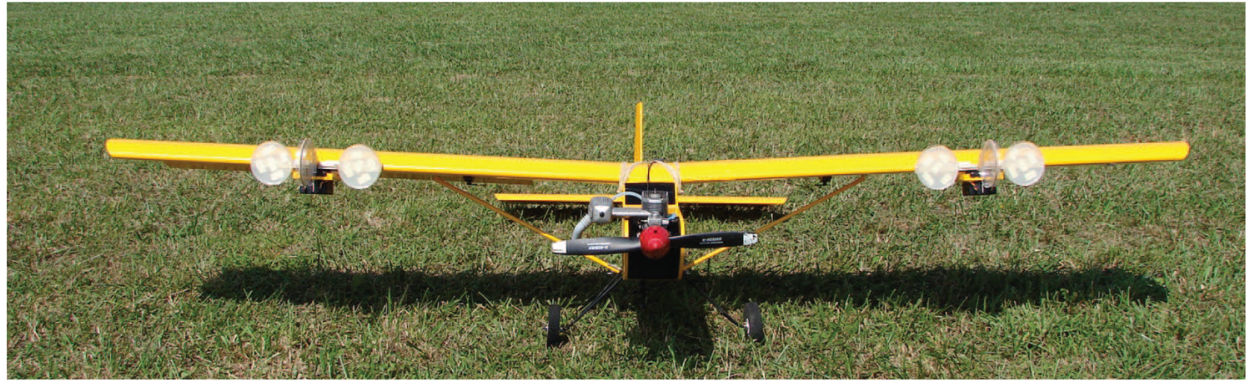

(a)

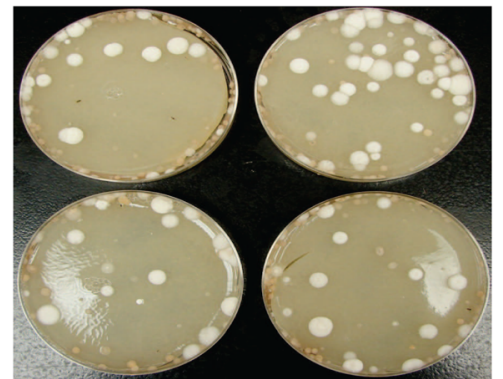

(b)

FIG. 3. (Color online) An autonomous UAV equipped with microbe-sampling devices for collecting Fusarium in the lower atmosphere (a). The sampling devices are closed during takeoff and landing and are opened once the UAV has reached its target sampling altitude. Petri plates with white colonies of $F u s a r$ ium cultured from an individual sampling mission are shown in (b). 
TABLE I. Parameters involved in estimating the aerial concentration of Fusarium spores.

\begin{tabular}{lcc}
\hline \hline Parameter & Value & Source \\
\hline $\mathrm{U}$ & $60.0 \pm 0.1 \mathrm{~km} / \mathrm{h}$ & GPS or onboard measurements \\
$\mathrm{G}$ & $9.81 \mathrm{~m} / \mathrm{s}^{2}$ & Physical constant \\
$\mathrm{v}_{\mathrm{s}}$ & $1.3 \mathrm{~mm} / \mathrm{s}$ & Schmale et al. (Ref. 49) \\
$\mathrm{T}_{\mathrm{f}}$ & $600-900 \mathrm{~s}$ & Onboard measurements \\
$\mathrm{N}$ & $4 \mathrm{or} 8$ & Number of petri plates on UAV \\
$\mathrm{R}$ & $4.5 \mathrm{~cm}$ & Radius of petri plate \\
$\mathrm{V}$ & $229 \mathrm{~m}^{3}$ & Derived parameter \\
$\mathrm{S}$ & 0.02454 & Derived parameter \\
$\mathrm{E}_{\mathrm{pp}}$ & $\approx 1.3 \%$ & Derived parameter \\
$\mathrm{C}$ & $0-18$ spores $/ \mathrm{m}^{3}$ & Derived parameter \\
\hline \hline
\end{tabular}

$$
C=\frac{N_{p}}{E_{p p} V}
$$

where $E_{p p}$ is an empirically determined efficiency of collection of spores in the petri plates,

$$
E_{p p}=\frac{0.594}{1+0.268 S^{-1.527}},
$$

and $S$ the Stokes number of the spores. The Stokes number of the spores is calculated using the formula $S=U \tau_{R} / 2 r$ where $U$ is the airflow speed in the free upstream approaching the sampler and $\tau_{R}=v_{s} / g$ is the particle relaxation time, where $v_{s}$ is the settling speed of the spores in still air and $g$ is the acceleration due to gravity. We used a value of $v_{s}=1.3$ $\mathrm{mm} / \mathrm{s}$ which is the average value of the experimentally determined range of settling speeds for a single species of Fusarium ( $F$. graminearum). ${ }^{48}$ The parameters needed for the concentration estimate and their range of values are summarized in Table I. The work by Prussin et al. ${ }^{50}$ showed that Fusarium species with different spore types will be collected with different sampling efficiencies. Future work aims to resolve the range of Fusarium species (and corresponding spore types) in our atmospheric collections to more accurately predict real (effective) atmospheric concentrations of Fusarium. The concentration $C$ is obtained as a number of spores per $\mathrm{m}^{3}$ of air.

Fig. 4 shows the concentration of Fusarium spores versus the flight number. Note that the flight number increases sequentially in time, but the flights were not evenly spaced in time. Flights were clustered around roughly week-long sampling campaigns, thus the time between any two flights could be as short as $1 \mathrm{~h}$ or as long as several months, and our main concern is fluctuations occurring on time-scales of significantly less than a day. Nevertheless, Fig. 4 is useful in showing that there are small fluctuations in the concentrations of Fusarium along with large and rapid fluctuations in the concentrations, i.e., sudden increases in observed concentrations of Fusarium, followed by sudden decreases in observed concentrations.

We are interested in an aerobiological transport framework to help explain these observations. To do this, we correct for the small fluctuations by setting a baseline concentration of $\bar{C}=3.350$ spores $/ \mathrm{m}^{3}$, which is the mean plus half the standard deviation of these samples. It is necessary to define a time-scale over which changes in concentration are regarded as punctuated changes. We choose two time-scales, 12 and $24 \mathrm{~h}$, to determine if the change in concentration is a punctuated change. Since most of the samples were collected during the daylight hours, the $12 \mathrm{~h}$ time-scale measures changes in concentration of atmospheric Fusarium on the same day. On the other hand, the $24 \mathrm{~h}$ time-scale is long enough to account for any periodic day-night variations in the concentration of spores in the atmosphere for the few samples that were collected late in the evening. Furthermore, fungal spores have a limited viability time-scale, which is on the order of a day, due to spore death by UV radiation

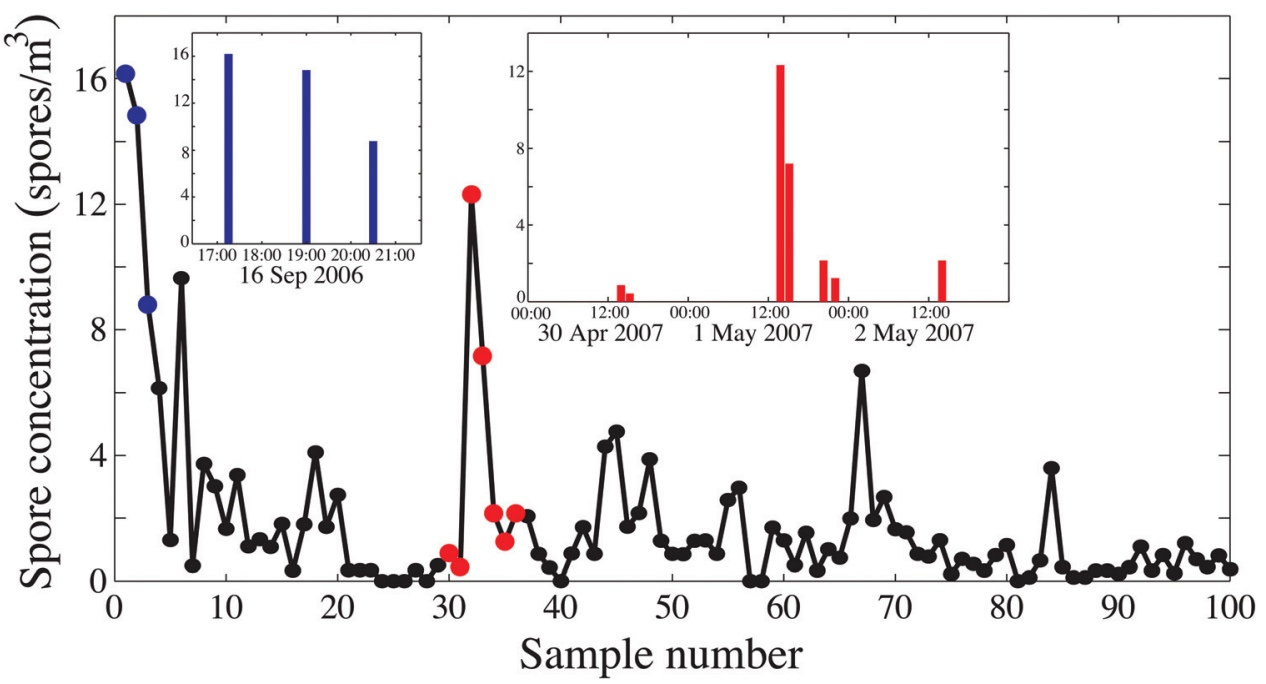

FIG. 4. (Color) Concentration of Fusarium spores (number $/ \mathrm{m}^{3}$ ) for samples from 100 flights conducted between August 2006 and March 2010 . The concentration numbers are the discrete points, the lines are merely to guide the eye. Note that the sample numbers on the horizontal axis are not evenly spaced in time. Examples of punctuated changes are shown in the insets. The left inset shows the concentrations, as well as the UTC times the samples were taken, on 16 Sep 2006; these correspond to the three large (dark blue) dots on the left. The right inset shows the UTC times of the sampled concentrations during several flights from 30 April 2007 to 2 May 2007, corresponding the large (red) dots. The sample collected at 14:00 UTC on 1 May 2007 contains the NIV strain. For both insets, no samples were collected between the times corresponding to the bars. 
exposure while airborne. Thus, considering time-scales of 12 and $24 \mathrm{~h}$ makes sense form both the atmospheric and biological standpoints. The choice of two time scales will also be a guide for future studies on scheduling sample collections. The calculated spore concentrations for 73 samples are shown in Tables IX and X in the Appendix.

An example of a punctuated change in the data is shown in the left inset of Fig. 4. After two very high concentration samples between 17:00 and 19:00 UTC on 16 Sep 2006, the concentration dropped by nearly one half by 20:30. Another example of punctuated changes is shown in the right inset of Fig. 4. The inset shows two punctuated changes, one a steep rise in the concentration of Fusarium from 15:00 UTC on 30 April 2007 to 14:00 UTC on 1 May 2007 and afterwards a rapid decrease in the concentration of spores at 20:15 UTC on 1 May 2007. This punctuated change is of particular interest because an analysis of specific strains of Fusarium observed in the sample collected at 14:00 showed the presence of a strain of $F$. graminearum that produced the mycotoxin nivalenol (NIV). ${ }^{50}$ Strains of $F$. graminearum that produce NIV have only been recovered from fields in North Carolina and New York, $100 \mathrm{~km}$ from our sampling location and, notably, have not been recovered from fields in Virginia. $^{50}$ Thus, in this specific case, we have punctuated changes in the atmospheric concentrations of members of the entire genus as well as strains of an individual species.

\section{B. Hypotheses on ATBs and punctuated changes}

The observed changes in the atmospheric concentrations of Fusarium suggest that we are sampling masses of air that differ not only in the relative amount of Fusarium, but also in their composition of different species and/or strains of Fusarium. We hypothesize that moving three-dimensional atmospheric LCSs are partitioning large-scale air masses, a small portion of which we are sampling with UAVs, as illustrated in Fig. 5.

On the meso- to synoptic-horizontal scale $(10-100 \mathrm{~km})$, the hypothesized situation is as illustrated in Fig. 6. The upper portion of the figure shows three time snapshots of the atmosphere (as seen from above and shown schematically), moving left to right in time. The thick (red) curves are atmospheric transport barriers (repelling LCSs) which, in this hypothetical example, bracket a set of air containing a high concentration of Fusarium, forming a moving boundary between the high concentration air mass and the neighboring low concentration ones. For the moment, we will merely assume this situation and not consider how it arises. Suffice it to say that filaments of distinct atmospheric composition sandwiched between LCS have been referred to in the literature. ${ }^{13-15,17,51,52}$ At time $t_{1}$, when the first sample is collected, only trace levels of Fusarium are detected. As the right-side LCS bounding this air mass moves over the sampling location, a sudden increase in Fusarium is detected, including at the sampling time $t_{2}$, followed later by another punctuated change as the left-side LCS boundary passes over. At $t_{3}$, once again only low levels of Fusarium are detected in the sampled air. This is one proposed mechanism to explain rapid changes in the composition of the air over a short interval of time, i.e., we are sampling either side of a repelling LCS.

Another alternative hypothesis to explain punctuated changes shown in Fig. 7 is using the attracting LCSs. The (locally) attracting LCSs act as a moving template around which microorganisms such as Fusarium will collect for relatively long periods of time (hours to days). Sets of air get stretched along attracting LCSs quickly. Whenever the air in the attracting LCS is sampled, it can be expected to harbor a distinct composition compared to the air on points that are on either side of it and sufficiently far from it. Thus, an alternative hypothesis is that a punctuated change is associated with the passage of an attracting LCS over the sampling point.

We summarize these two hypotheses below.

$\mathrm{H}_{11}$ : Punctuated changes in the atmospheric concentration of Fusarium imply the movement of a repelling LCS over the sampling point, between the two sampling times.

$\mathrm{H}_{12}$ : Punctuated changes in the atmospheric concentration of Fusarium imply the movement of an attracting LCS over the sampling point, between two

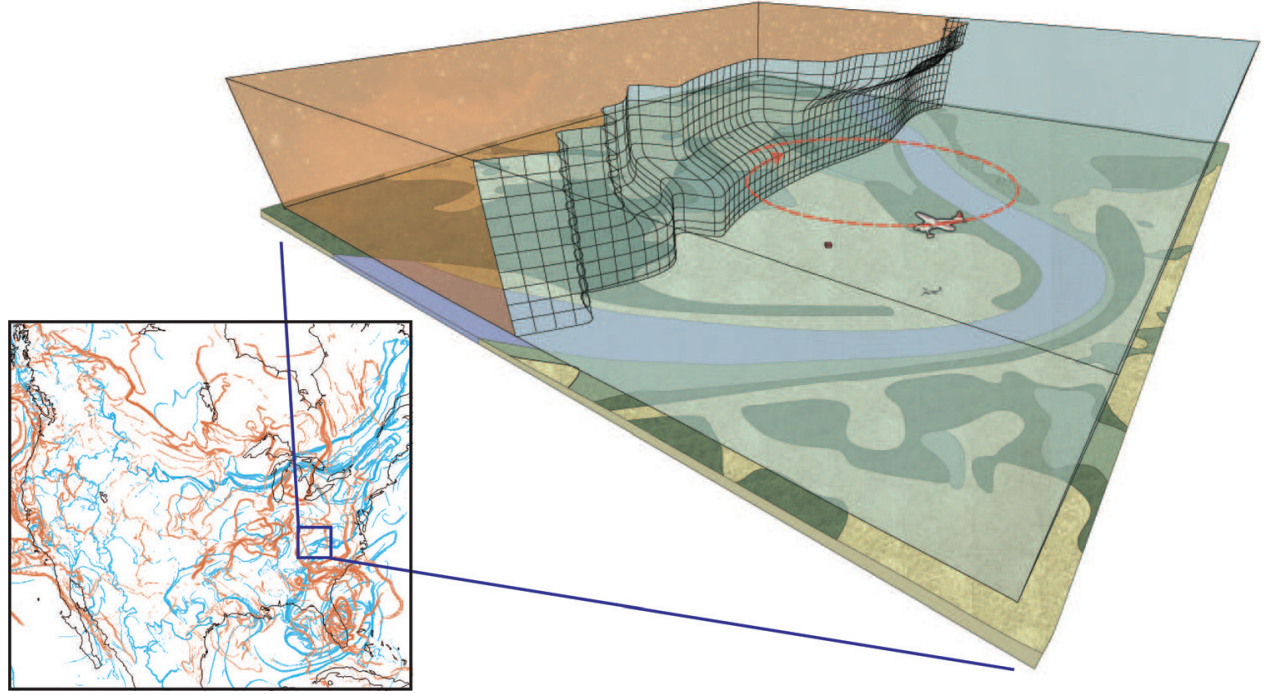

FIG. 5. (Color) Atmospheric LCS are large-scale features with local effects. An LCS (shown as undulating mesh) separates two volumes of air as it moves over topography, shown schematically on a scale of several kilometers near our sampling site at Virginia Tech's Kentland Farm in Blacksburg, VA. Autonomous UAVs are used to collect Fusarium on both sides of an LCS. We hypothesize that mixed populations of Fusarium in the lower atmosphere are mediated by LCS, as an LCS is a highly repelling material surface separating two relatively well-mixed air masses. This LCS is just one of a large-scale network of repelling and attracting LCS on the continental scale (left). 


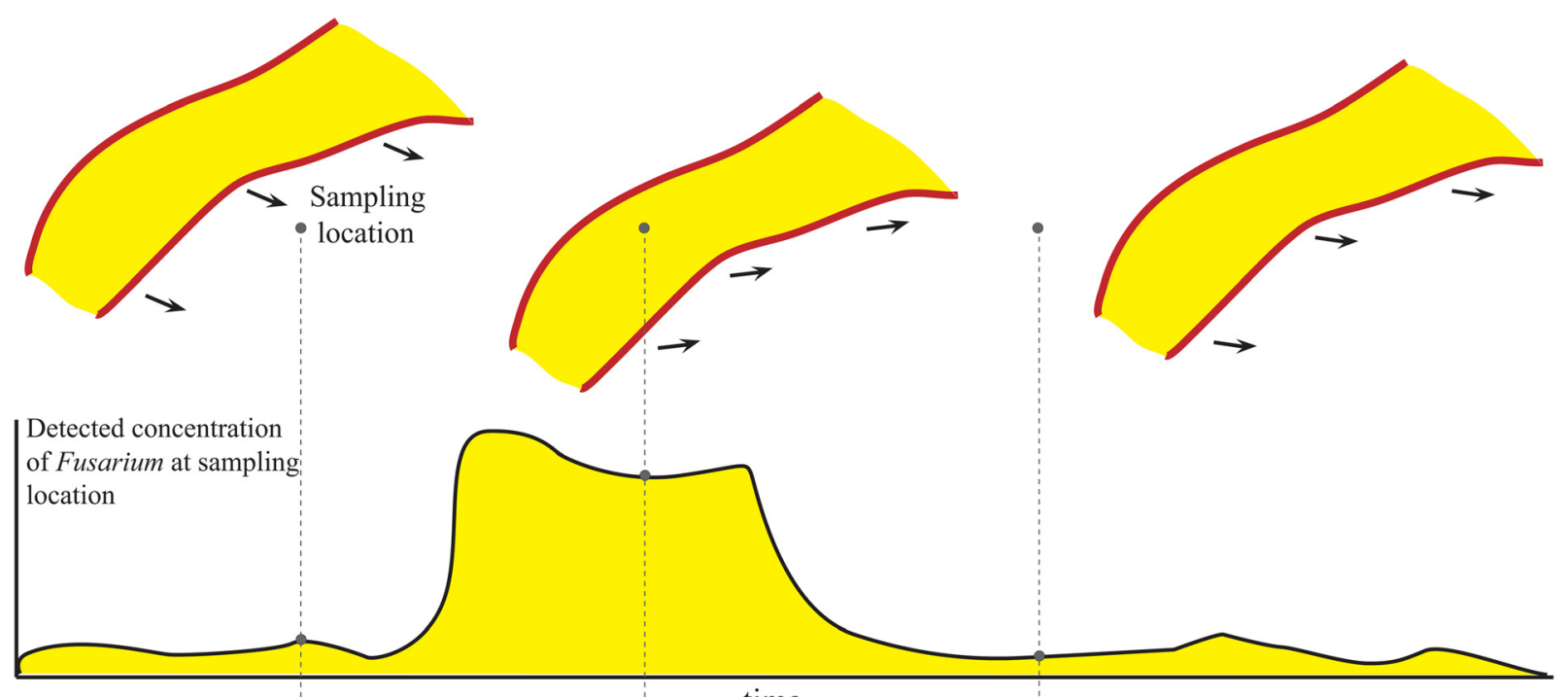

time

FIG. 6. (Color online) Movement of a "cloud" of relatively high concentration of Fusarium bracketed by repelling LCSs (upper panel, three time snapshots going from left to right) and the corresponding abrupt changes in detected concentrations of Fusarium at a geographically fixed sampling location (below). The continuous signal with time is hypothetical. Sampling yields only a discrete number of observations, as sampling takes a finite duration and the time between samples can be significant. In this example, two punctuated changes are shown (an abrupt increase followed by an abrupt decrease), which are a consequence of the particular LCS movement with respect to the sampling location.

sampling times or by the presence of an attracting LCS over the sampling point at the sampling time of the punctuated change.

We may want to consider, instead of each of these in isolation, a hypothesis merging the two, as follows:

$\mathrm{H}_{1}$ : Punctuated changes in the atmospheric concentration of Fusarium imply the movement of an atmospheric transport barrier (attracting or repelling LCS) over the sampling point, between the two sampling times.

The null hypothesis is that the movement of the repelling (attracting) LCS is not correlated with the punctuated changes in the concentration of Fusarium.

$\mathrm{H}_{0}$ : Punctuated changes in the atmospheric concentration of Fusarium do not imply the movement of an atmospheric transport barrier (attracting or repelling $L C S$ ) over the sampling point between the two sampling times.

The hypotheses $\mathrm{H}_{11}, \mathrm{H}_{12}$, and $\mathrm{H}_{1}$ only say that the punctuated changes imply the passage of an LCS and not the converse.

TABLE II. Contingency table.

\begin{tabular}{lcc}
\hline \hline & \multicolumn{2}{c}{ Punctuated change has occurred $\left(\Delta C_{p}\right)$} \\
\cline { 2 - 3 } LCS passed over & Yes & No \\
Kentland farm (L) & $n_{1}$ & $n_{2}$ \\
\hline Yes & $n_{3}$ & $n_{4}$ \\
No & \\
\hline
\end{tabular}

These hypotheses are meaningful only if the number of LCSs passing the geographically fixed sampling location, i.e., the sampling point in a given interval of time is not very high. For example, if we find that there are five LCSs passing the sampling point every hour, then one could easily associate every punctuated change with the movement of an LCS. Therefore, we measured the time interval, $\Delta t$, between the passage of consecutive LCSs passing the sampling point over a period of $591 \mathrm{~h}$ covering the sampling times and spread over roughly three years. The time interval $\Delta t$ depends on how LCS are defined. These details are provided in Sec. IV. In Fig. 8, we plot the distribution of $\Delta t$. The mean

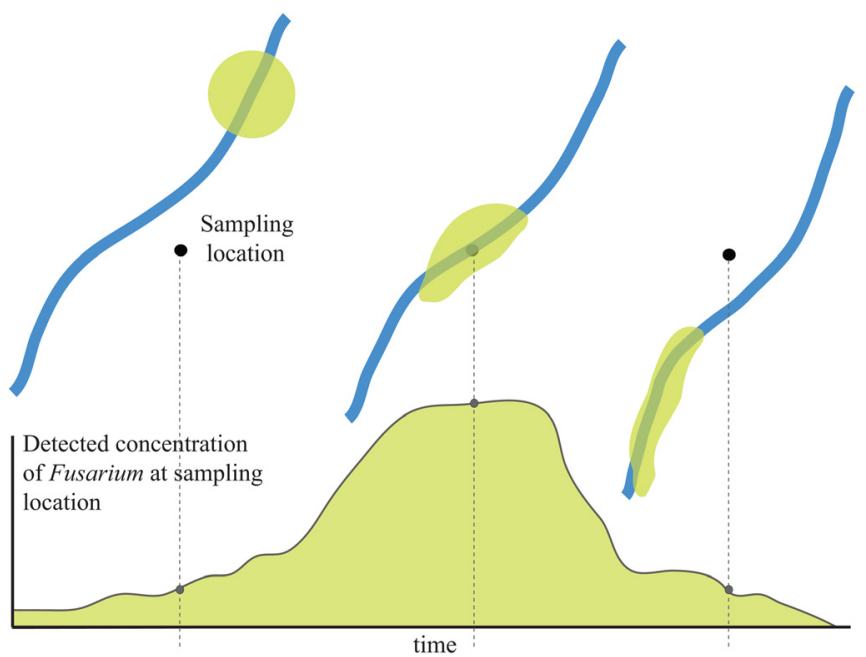

FIG. 7. (Color online) Movement of a "cloud" of relatively high concentration of Fusarium along an attracting LCSs (upper panel, three time snapshots going from left to right) and the corresponding abrupt changes in detected concentrations of Fusarium at a geographically fixed sampling location (below). The continuous signal with time is hypothetical. Sampling yields only a discrete number of observations, as sampling takes a finite duration and the time between samples can be significant. 


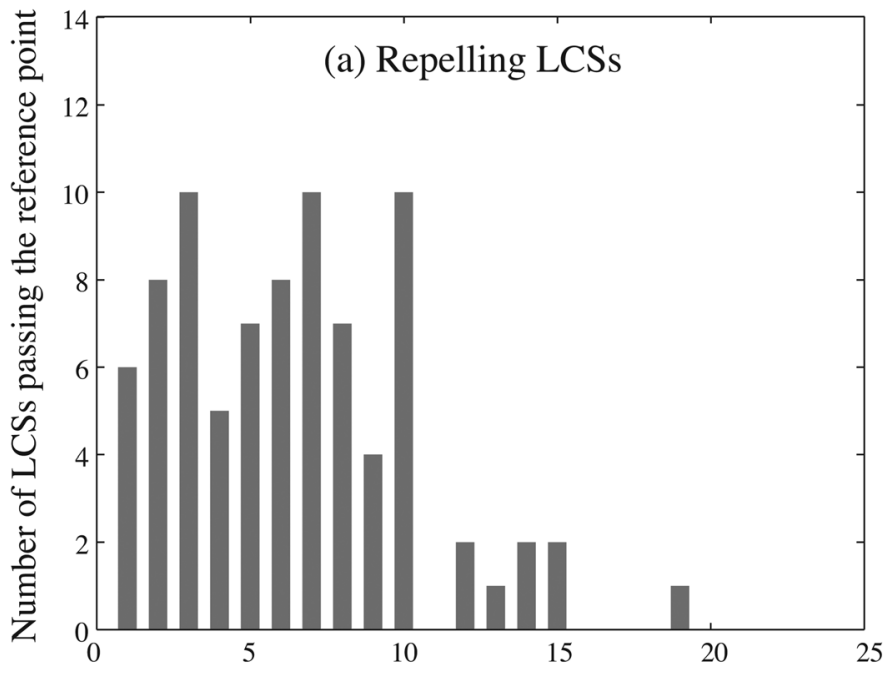

Time interval between passage of LCSs (hours)

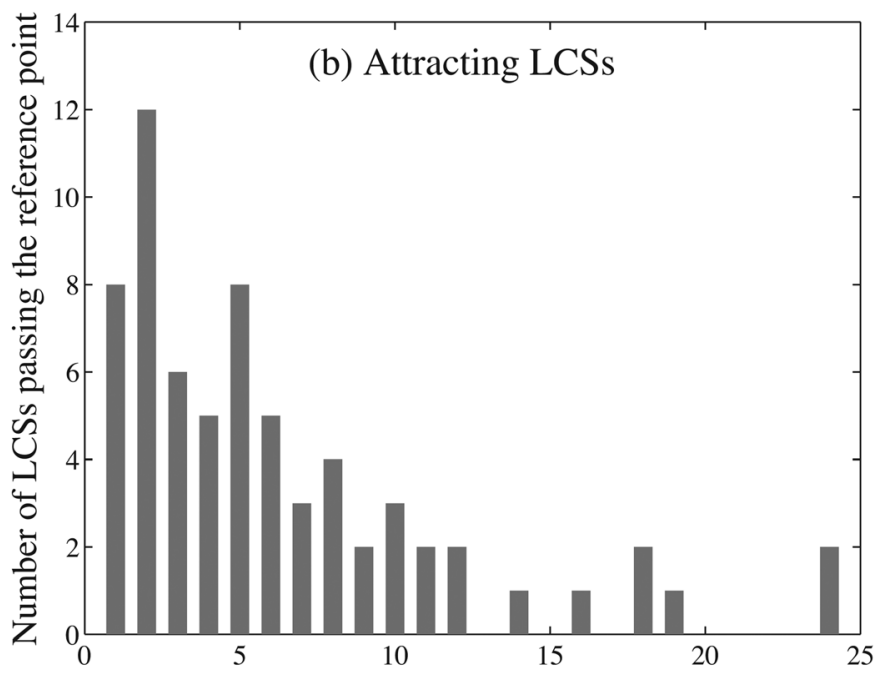

Time interval between passage of LCSs (hours)

FIG. 8. The relative distribution of $\Delta t$, the time intervals between consecutive LCS passages over the sampling point for (a) repelling LCSs and (b) attracting LCSs.

$\Delta t$ is $7.11 \mathrm{~h}$ for repelling LCS and $8.817 \mathrm{~h}$ for attracting LCS. Thus, the small number of LCSs passing the reference point ensures that this is not a very common event by itself. From Fig. 8, we can see that the highest number of LCS is clustered around small time intervals. However, this is because often a cluster of LCSs pass the reference point in quick succession. The example in Sec. V illustrates this. Moreover, many of these closely spaced LCS are associated with the punctuated changes in the concentration of atmospheric Fusarium. It should be noted that the distribution of $\Delta t$ is dependent on the choice of the threshold value of the FTLE which defines the LCS, i.e., we only consider locations where $\sigma>\sigma_{\text {min }}$, where the choice of $\sigma_{\text {min }}$ is discussed further in Sec. IV to correspond with the scales of interest. A lower value of $\sigma_{\min }$ would produce shorter $\Delta t$ 's.

\section{Statistical framework for hypotheses testing}

The concentration of samples was ordered in time from $t_{0}$ to $t_{N-1}$, with corresponding concentrations $C_{0}$ to $C_{N-1}$. We considered concentration changes only between two consecutive times separated by less than a specified number of hours $T$, i.e., we required $t_{k}-t_{k-1}<T$, considering two cases: $T=24 \mathrm{~h}$ and $T=12 \mathrm{~h}$. The concentration change over that time was calculated as $\Delta C_{k}=C_{k}-C_{k-1}$, i.e., $\Delta C_{k}$ is the change in concentration over the $k$ th time interval. We can categorize each of the change in concentrations, $\Delta C_{k}$ as (1) a punctuated change or (2) not a punctuated change. For each change in concentration, we can determine whether a repelling or attracting LCS has passed the sampling point at Kentland farm, in the time interval $\left(t_{k-1}, t_{k}\right)$, or if an attracting LCS is hovering on the sampling point during the same time interval. Therefore, our variables are categorical. A categorical variable is one for which the measurement scale consists of a set of categories. ${ }^{53}$ In our case, this set of categories consists of two values, $\{Y e s, N o\}$. The data and categorical variables for each consecutive pair of observations are given in Tables IX and X of the Appendix.
Contingency tables are a good way to describe bivariate categorical data. ${ }^{53}$ To explain the use of contingency tables and correlation, we first define our variables precisely. Our random variables are $\Delta C_{P}$, a punctuated change in concentration, and $L$, the passage of a transport barrier over our sampling point at Kentland farm. These variables can take the categorical values Yes or No. The correlation between the two variables can be studied by the $2 \times 2$ contingency table shown in Table II.

The statistical correlation between the two tables can be measured by the $\phi$ coefficient defined by

$$
\phi=\frac{n_{1} n_{4}-n_{2} n_{3}}{\sqrt{\left(n_{1}+n_{2}\right)\left(n_{3}+n_{4}\right)\left(n_{2}+n_{4}\right)\left(n_{1}+n_{3}\right)}}
$$

if the denominator is not zero, with $n$ 's as in Table II. If any of the sums in the denominator of Eq. (10) are zero, then $\phi$ is defined to be 0 , i.e., the variables are statistically independent. The $\phi$-coefficient measures two-way correlation, however, we hypothesized only a one-way correlation. Hence, we do not expect the value of $\phi$ to be close to 1 . To verify our one-way hypothesis, we use the specificity $\left(s_{1}\right)$ and sensitivity $\left(s_{2}\right)$. The specificity $s_{1}$ is defined as

$$
s_{1}=\frac{n_{4}}{n_{4}+n_{2}} \text {. }
$$

A specificity of 1 means that the LCS diagnostic test does not produce any false positives, i.e., the test is very specific. The sensitivity $s_{2}$ of a test is defined as

$$
s_{2}=\frac{n_{1}}{n_{1}+n_{3}} .
$$

A sensitivity of 1 means that the LCS diagnostic test identifies all punctuated changes in the concentration of atmospheric Fusarium.

To determine significance, we calculate the probability that the correlations of LCS passages and punctuated 


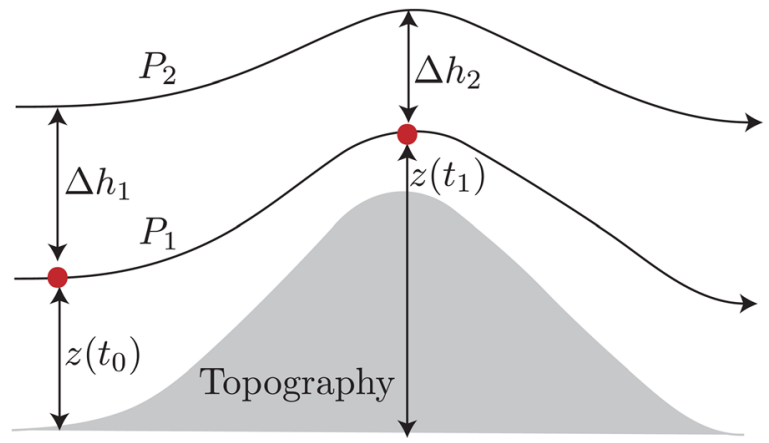

FIG. 9. (Color online) Vertical movement of an air mass along an isobaric surface.

changes could have been purely accidental. Formally, this is given by the $p$-value obtained from Fischer's exact test. ${ }^{53}$ The $p$-value is given by the formula,

$$
p=\sum_{k} \frac{\left(n_{1}+n_{2}\right) !\left(n_{3}+n_{4}\right) !\left(n_{1}+n_{3}\right) !\left(n_{2}+n_{4}\right) !}{n_{1} ! n_{2} ! n_{3} ! n_{4} !\left(n_{1}+n_{2}+n_{3}+n_{4}\right) !}
$$

where the summation is over all the favorable cases that are at least as extreme the observed data, holding the marginal totals $n_{1}+n_{2}, n_{3}+n_{4}, n_{1}+n_{3}$, and $n_{2}+n_{4}$ constant. The $p$ value is the probability of obtaining the data if the null hypothesis, $\mathrm{H}_{0}$, is true. The critical $p$-value below which the results are statistically significant can be considered to be 0.05. A low $p$-value combined with a high sensitivity and specificity will allow us to reject the null hypothesis.

We present the resulting contingency tables and statistical measures in Sec. V, after a discussion of the computation of LCS.

\section{COMPUTATION OF LCS}

For computing the trajectories of parcels of air, we used the WRF NAM-218 velocity data set provided by NOAA. ${ }^{54}$ The data consist of horizontal velocity specified on a grid of points spaced at intervals of approximately $12.5 \mathrm{~km}$ over North America using the Lambert conformal projection. The positive $x$ direction is towards the east and the positive $y$ direction is to the north. We take an initial grid of virtual particles (air parcels) with a uniform spacing of $5 \mathrm{~km}$ covering a grid area of $2000 \mathrm{~km} \times 2000 \mathrm{~km}$ centered around our sampling point, on a $900 \mathrm{mb}$ pressure surface. The $900 \mathrm{mb}$ pressure surface was chosen as it roughly corresponds with the pressure height of the sampling location of the UAVs $(100 \mathrm{~m}$ above ground level at Kentland Farm). These particles are advected using a RK4 algorithm with a relative tolerance of $10^{-6}$. The size of the time step varies adaptively between half a minute to $10 \mathrm{~min}$. To integrate the velocities and find the trajectories, we need a smoothly defined velocity field. The velocity data we have are defined only every $3 \mathrm{~h}$ and at discrete points spaced at $12.5 \mathrm{~km}$ intervals. Therefore, we interpolate the data both temporally and spatially. To this end, we use a bicubic interpolation spatially to find the velocity in the interior of a cell defined by the NAM-218 data grid points and a cubic interpolation temporally to find the velocity between the $3 \mathrm{~h}$ intervals. We used these specific interpolation methods since they produce a continuously differentiable velocity field, while a linear interpolation may not produce a smooth velocity field. Bicubic and tricubic interpolation methods have been used in earlier works by Lekien and others. ${ }^{12,32,55}$

In performing these computations, we made the assumption that the flow of air in the atmosphere is almost on surfaces of constant pressure under quasi-geostrophic conditions ${ }^{22}$ and that the flow on nearby isobaric surfaces does not vary significantly. Flow on an isobaric surface does not preclude the vertical movement of air. As shown in Fig. 9, isobaric surfaces themselves move vertically. From the NAM data set, we found that in most of eastern North America, the vertical velocity is \pm 0.01 to $\pm 0.03 \mathrm{~Pa} / \mathrm{s}$. This means that, in a "worst-case" scenario, an air mass can move up or down by a pressure height of $8.6 \mathrm{mb}-25.9 \mathrm{mb}$ in $24 \mathrm{~h}$. However, in general, sustained vertical velocities in one direction do not persist for $24 \mathrm{~h}$ along a trajectory and tend to balance out.

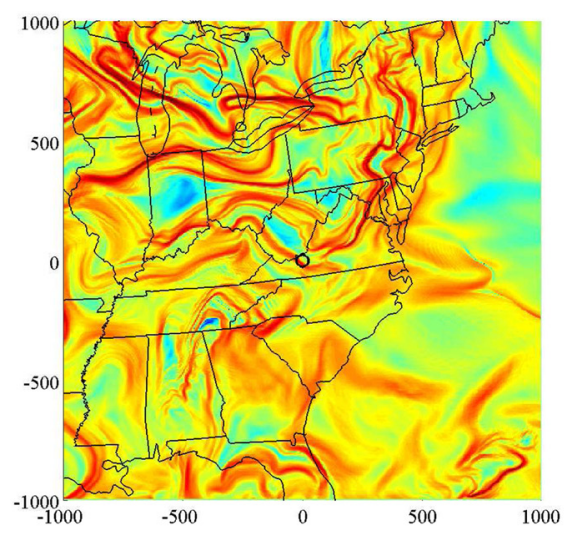

(a) $875 \mathrm{mb}$

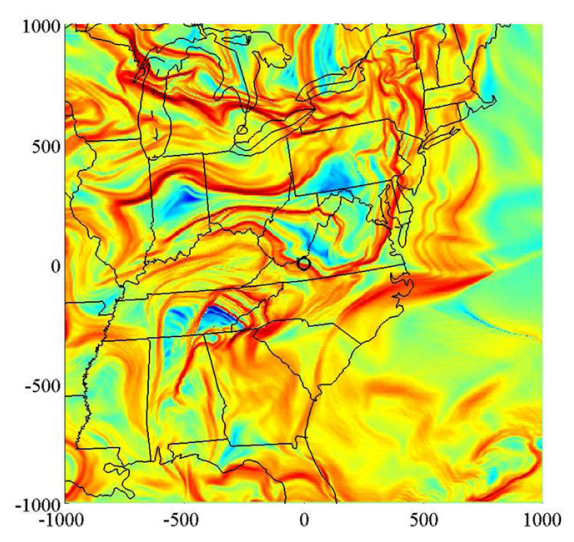

(b) $900 \mathrm{mb}$

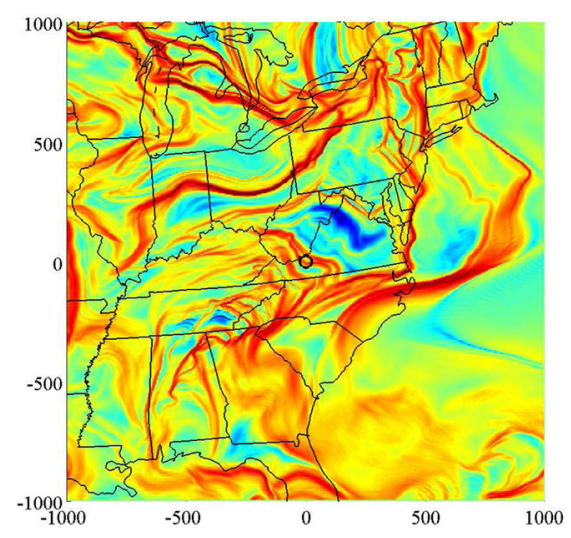

(b) $925 \mathrm{mb}$

FIG. 10. (Color online) FTLE computations are insensitive to small finite changes in the initial pressure. The forward time FTLE ( $T=24$ h) is qualitatively the same on the (a) $875 \mathrm{mb}$, (b) $900 \mathrm{mb}$, and (c) $925 \mathrm{mb}$ isobaric surfaces at 12:00 UTC on 1 May 2007. The $x$ - and $y$-axis are in kilometers with the origin centered on the sampling location, Kentland Farm, shown by a circle in the center of the figure. Regions with a high FTLE value are shown in red (appear as ridges), while those with a low or zero FTLE value are in blue. 


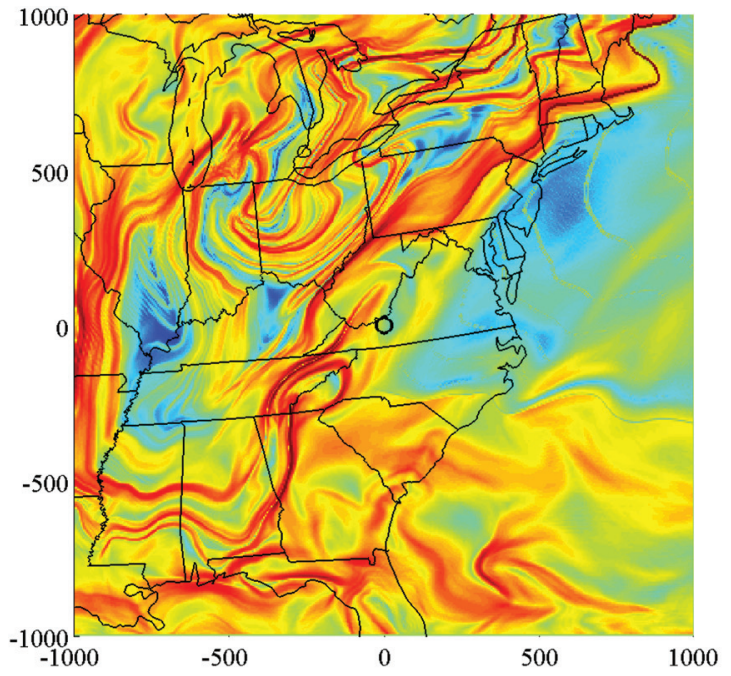

(a)

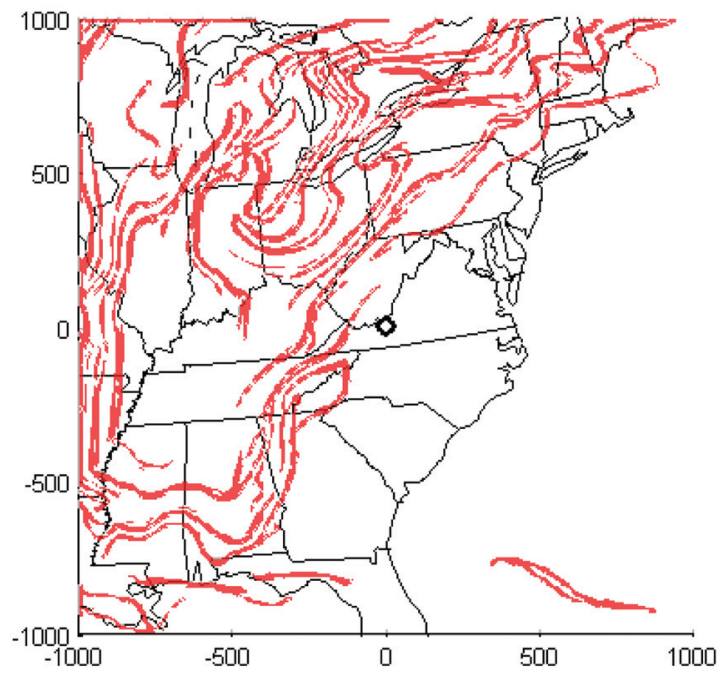

(b)

FIG. 11. (Color) (a) Sample FTLE field over the eastern United State at 21:00 UTC on 15 May 2007. The $x$ - and $y$-axis are the same as in Fig. 10. (b) Ridges extracted from the FTLE field in (a).

Since an air mass can rise or fall by about $25 \mathrm{mb}$, we calculated the FTLE field on three isobaric surfaces $(875 \mathrm{mb}$, $900 \mathrm{mb}$, and $925 \mathrm{mb}$ ) shown in Fig. 10 and find that while the FTLE fields of $900 \mathrm{mb}$ and $925 \mathrm{mb}$ are qualitatively similar with the FTLE field on the $875 \mathrm{mb}$ surface being slightly different. We observe two main differences between the raw FTLE fields are that for a given integration time, air particles do not separate as rapidly on a higher isobaric surface, i.e., ridges in the FTLE field are of a lower height. Second, the FTLE ridges themselves are at slightly different positions due to the shear between the different isobaric layers. We find that horizontal separation of trajectories due to small initial differences in height is primarily through shear at a reference pressure of $900 \mathrm{mb}$. This means that while the terms $\frac{\partial \phi_{1}}{\partial x_{3}}$ and $\frac{\partial \phi_{2}}{\partial x_{3}}$ in Eq. (6) may have a high magnitude, but they are due to shear. This provides further justification for the use of partial FTLE to identify barriers to horizontal transport in the atmosphere. We note that the simplified analysis using isobaric atmospheric flow is a first step towards the study of transport barriers and punctuated changes in a 3-dimensional atmospheric flow.

An integration time of $24 \mathrm{~h}$ was chosen for two reasons: (1) to account for the effects of the day-night cycle in the velocity field and (2) to satisfy the approximation that the air flow is on isobaric surfaces. The FTLE field $\sigma(x, y)$ obtained by advecting particles for $24 \mathrm{~h}$ will rule out any influence of a day-night cycle and satisfy the approximation of isobaric flow. For specific cases, where the FTLE field is not smooth enough, we used a finer grid resolution of $1 \mathrm{~km}$. We also verified our FTLE computations using the software"Newman."

The FTLE field is obtained by finite differences. Technically, this is a partial FTLE field (see Eq. (7)) since we are ignoring any sensitivity to variation of the initial height of particles. However, due to the vertical rigidity of the atmospheric flow, this sensitivity to variations in initial height is negligible one the time-scale of $24 \mathrm{~h}$. Fig. 11(a) shows a sample FTLE field obtained for particles starting at 21:00 UTC on 15 May 2007 and integrated for $24 \mathrm{~h}$ on the $900 \mathrm{mb}$ pressure surface. The regions of high FTLE are shown in red. The ridges in the FTLE field in this case cannot be obtained by just setting a threshold for the value of $\sigma$. Moreover, the FTLE field has many small ridges which are not significant as transport barriers on large spatial scales.

To overcome these issues, we used a heuristic algorithm that uses an intuitive geometric definition of ridges. Assuming that the principal curvatures at every point in the FTLE field are given by $\kappa_{1}(x, y)$ and $\kappa_{2}(x, y)$ with $\kappa_{1}>\kappa_{2}$, we used three criteria to determine if a point $(x, y)$ in the FTLE field lies on a ridge.

$$
\begin{gathered}
\sigma(x, y) \geq \sigma_{\text {min }}>0, \quad \kappa_{2}(x, y)<0, \\
\kappa_{1}(x, y)+\kappa_{2}(x, y)<0 .
\end{gathered}
$$

The second condition says that the mean curvature should be negative, i.e., the saddle surface should be more concave than convex. We used $\sigma_{\min }=0.04 \mathrm{hr}^{-1}$ for which a line element of length $\delta l_{0}=5 \mathrm{~km}$ would, over $T=24 \mathrm{~h}$, be stretched to a length of $\delta l=e^{\sigma}{ }_{\min }^{T} \delta l_{0} \approx 13 \mathrm{~km}$ which is about the grid spacing for velocity of the NAM data set. In practice, the ridge values are quite a bit higher than $\sigma_{\min }$, usually several times higher. As an example, we applied the heuristic criteria on ridge curvature and height to the sample FTLE field shown in Fig. 11(a) to extract the FTLE ridges as shown in (b). The ridges in the FTLE field in our computations have a finite thickness. This is expected, since boundaries with zero thickness that persist for several hours are unrealistic in the atmosphere. The thickness of the ridges extracted from the FTLE field depends on the initial spacing of points in the grid. The thickness of the ridges is at least twice the grid spacing in our computations. However, it is unclear if the thickness of the ridges always converges to zero by taking finer grid spacing.

To test the first hypothesis, $\mathrm{H}_{11}$, we check if a repelling LCS passes over the sampling point between consecutive samplings. To test the second hypothesis, $\mathrm{H}_{12}$, we also need to check if an attracting LCS exists over the sampling point at the precise instant of sampling. This second condition is 


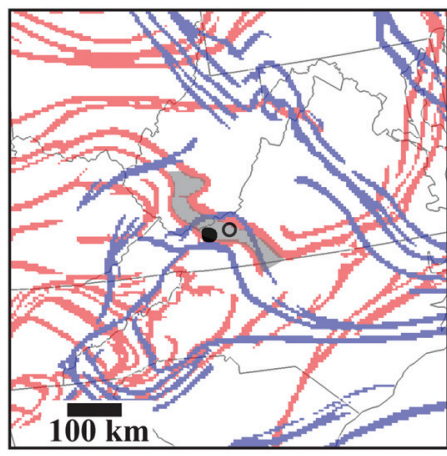

(a)

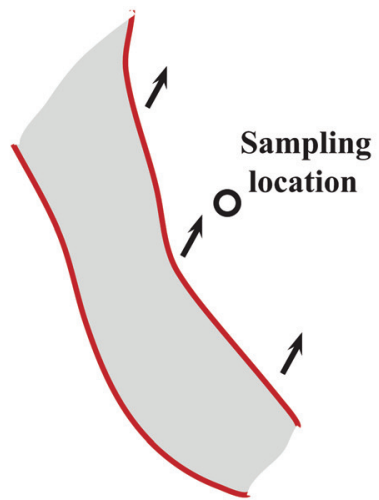

(d)

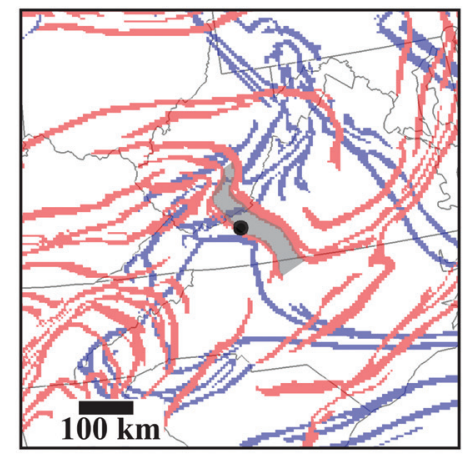

(b)

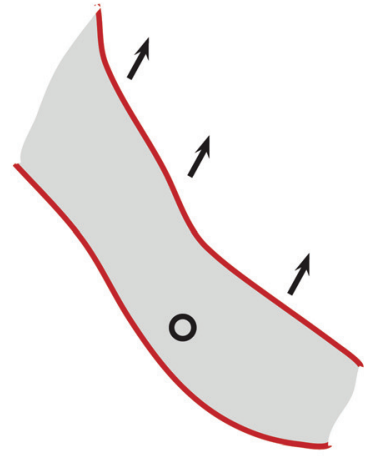

(e)

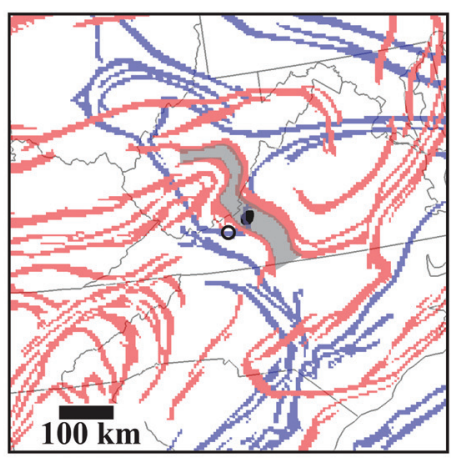

(c)

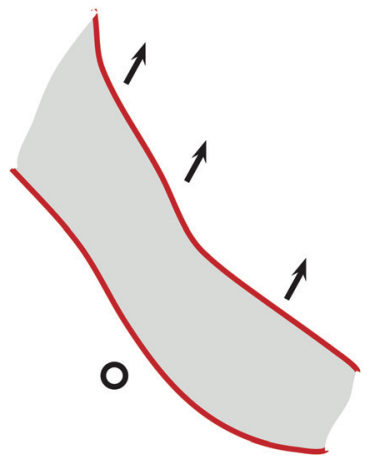

(f)

FIG. 12. (Color) LCSs (repelling LCS shown in red and attracting LCSs shown in blue) on the $900 \mathrm{mb}$ surface for the time surrounding the "spike" in concentration at 14:00-15:00 UTC on 1 May 2007. (a) 12:00 UTC on 1 May 2007, (b) 15:00 UTC on 1 May 2007, and (c) 18:00 UTC on 1 May 2007. The gray region represents a hypothesized air mass of high Fusarium spore concentration "sandwiched" between two repelling LCSs. The corresponding schematic is shown below in (d)-(f) (cf. Fig. 6). The open circle is the sampling location at Kentland Farm. The filled circle in (a)-(c) represents the portion of the air mass sampled at 15:00 UTC on 1 May 2007.

unrealistic to verify because of small uncertainties in the velocity data and interpolations. Therefore, we made two simplifications. First, we tracked the position of attracting and repelling LCSs at $15 \mathrm{~min}$ intervals. This provides the needed temporal resolution as sampling events are typically $15 \mathrm{~min}$ long. Second, we represented our sampling station not by a single point, but by a circle with a diameter of $10 \mathrm{~km}$ which is of the order of the data grid size and the thickness of the ridges in the FTLE field. An LCS is considered to have passed the sampling point at Kentland farm within these 15 min if it lies on either side of the center of the circle or in the interior and exterior of the circle at the end points of the time interval. Attracting LCS that is passing through any point in the circle at the sampling time is regarded to pass through

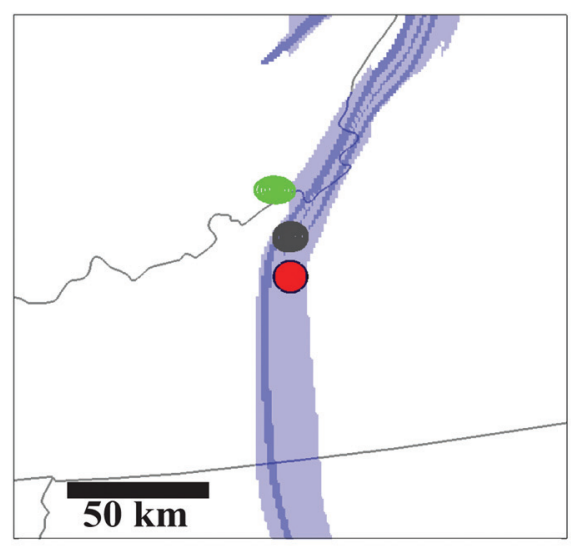

(a)

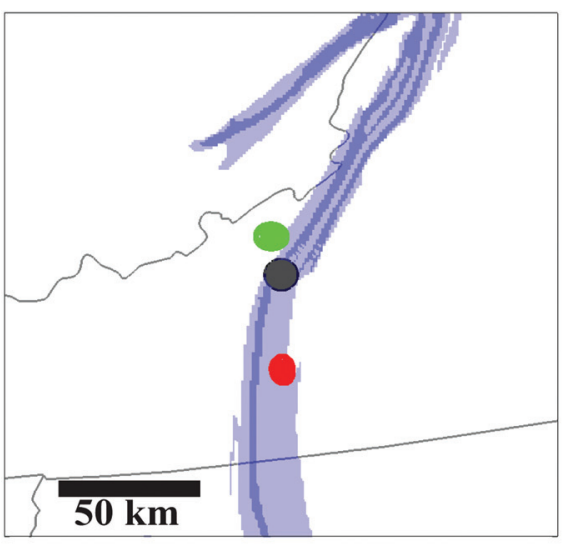

(b)

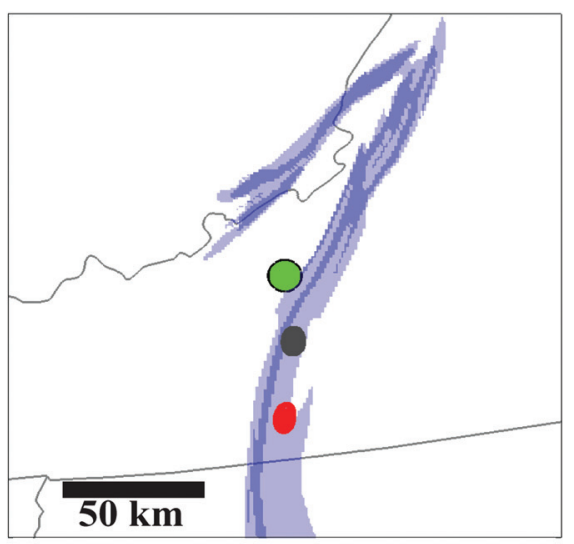

(c)

FIG. 13. (Color) The thick blue curve in the figure is an attracting LCS, the thin curves embedded within this attracting LCS are the sharpest ridges in the FTLE field. The red, grey, and green sets indicate air sampled at (a) 17:15, (b) 19:00, and (c) 20:30 UTC, respectively. When the attracting LCS passes over the sampling point, this Fusarium laden air mass is sampled twice, at 17:15 UTC and 19:00 UTC. After the attracting LCS moves past the sampling point, the air mass sampled at 20:30 UTC (green colored set) has a low concentration of Fusarium. Note, this figure is on a smaller scale than Fig. 12. 
TABLE III. Contingency table for hypothesis $\mathrm{H}_{11}$ for $24 \mathrm{~h}$ time scale.

\begin{tabular}{lcc}
\hline \hline & \multicolumn{2}{c}{ Punctuated change has occurred $\left(\Delta C_{p}\right)$} \\
\cline { 2 - 3 } Repelling LCS passed over & Yes & No \\
Kentland farm (L) & 11 & 14 \\
\hline Yes & 5 & 43 \\
No & & \\
\hline \hline
\end{tabular}

the sampling point at Kentland farm. These two simplifications are also motivated by the fact that the atmospheric LCS has a finite thickness. At a representative speed of $10 \mathrm{~m} / \mathrm{s}$, points on an LCS can travel about $9 \mathrm{~km}$ in 15 mint which is about the diameter of the sampling circle. The time interval is, therefore, necessary and sufficient enough to track the movement of LCS into or out of the sampling circle. The results of LCS passage over the sampling circle preceding, during and after the sampling are shown in Tables IX and X.

\section{RESULTS}

Detailed results are shown here on the movement of LCSs associated with punctuated changes for the two sample sets shown in the insets of Fig. 4. Consider first the right inset, covering the 30 April 2007 to 2 May 2007 time frame. Concentrations of Fusarium on 30 April 2007 at 14:00 and 15:00 UTC were approximately 0.8 and 0.4 spores $/ \mathrm{m}^{3}$, respectively, which are within the normal range of the concentration of atmospheric Fusarium. Further the samples taken on 30 April 2007 did not contain strains of Fusarium graminearum that produce the mycotoxin NIV. ${ }^{50}$ The samples collected on 1 May 2007 at 14:00 and 15:00 UTC showed Fusarium concentration of 12.3 spores $/ \mathrm{m}^{3}$ and 7.2 spores $/ \mathrm{m}^{3}$, respectively. Moreover, the sample collected at 14:00 UTC contained a strain of $F$. graminearum that produced NIV. ${ }^{50}$ Further sampling at 20:15 UTC showed a $F u$ sarium concentration of only 2.1 spores $/ \mathrm{m}^{3}$. We suggest these punctuated changes in the concentration of Fusarium can be explained by the action of repelling LCSs (hypothesis $\mathrm{H}_{11}$ ), as shown schematically in Fig. 6.

Fig. 12 shows the atmospheric LCSs present during the time surrounding the "spike" in concentration around 14:0015:00 on 1 May 2007, with repelling LCS in red and attracting LCS in blue. Air masses of significantly different concentration of Fusarium are sandwiched between two closely spaced repelling LCSs. The passage of these LCS one after the other in quick succession is associated with the punctuated changes and hence the "spike". The details of this punctuated change are reported in more detail in Ref. [50]. We note that there are other weak attracting and repelling LCSs between the two strong repelling LCS, which were filtered

TABLE IV. Contingency table for hypothesis $\mathrm{H}_{12}$ for $24 \mathrm{~h}$ time scale.

\begin{tabular}{lcc}
\hline \hline & \multicolumn{2}{c}{ Punctuated change has occurred $\left(\Delta C_{p}\right)$} \\
\cline { 2 - 3 } Attracting LCS passed over & Yes & No \\
Kentland farm (L) & 6 & 16 \\
Yes & 10 & 41 \\
No & & \\
\hline
\end{tabular}

TABLE V. Contingency table for hypothesis $\mathrm{H}_{1}$ for $24 \mathrm{~h}$ time scale.

\begin{tabular}{lcc}
\hline \hline & \multicolumn{2}{c}{ Punctuated change has occurred $\left(\Delta C_{p}\right)$} \\
\cline { 2 - 3 } Attracting or repelling LCS passed & Yes & No \\
\cline { 2 - 3 } over Kentland farm (L) & 13 & 23 \\
Yes & 3 & 34 \\
No & & \\
\hline \hline
\end{tabular}

out. In our computations, we observe small filaments and lobes formed by weak repelling and attracting LCSs which are ubiquitous in the atmosphere. While we ignore these small scale structures as transport barriers, we note that these are responsible for mixing and homogenizing the composition of air between the large scale LCSs. ${ }^{56}$

A punctuated change that we suggest can be explained by hypothesis $\mathrm{H}_{12}$ occurred on 16 September 2006, the sample data for which was shown in Fig. 4, left inset. The air sample collected on 16 September 2006 at 17:15 and 19:00 UTC had a Fusarium concentration of 16.2 and 14.8 spores $/ \mathrm{m}^{3}$. The three sets that were sampled at these times are shown in Fig. 13. In this case, the attracting LCS acts as an atmospheric highway along which Fusarium is transported along. Thus, in Figs. 13(a) and 13(b), when the attracting LCS is on the sampling point the sampled air contains a high concentration of Fusarium, and once the attracting LCS moves away from the sampling point, the sampled air has a low concentration of Fusarium. Compare with the schematic shown in Fig. 7.

Similar to the computations for the preceding two cases, attracting and repelling LCSs were computed on a $900 \mathrm{mb}$ pressure surface for time intervals surrounding the 73 samples (Tables IX and X) which met the criteria for our analyses. The spore concentration calculations and the determination of punctuated changes for these samples are shown in the Appendix. Of the 73 samples, 16 show punctuated changes in the concentration of Fusarium on a $24 \mathrm{~h}$ timescale. We used the contingency tables to test each of our hypotheses $\mathrm{H}_{11}, \mathrm{H}_{12}$, and $\mathrm{H}_{1}$. The contingency table for testing hypothesis $\mathrm{H}_{11}$ is shown in Table III. The $p$-value for the data in Table IV corresponding to the hypothesis $\mathrm{H}_{11}$ on a 24 $\mathrm{h}$ time scale is 0.0017 . The sensitivity of the test for $\mathrm{H}_{11}$ is 0.6875 and the specificity of the test is 0.7544 . From this we can infer that punctuated changes were significantly associated with the movement of a repelling LCS. The correlation between the repelling LCS and punctuated changes using Eq. (10) is $\phi_{11}=0.3852$. The low two-way correlation $\phi_{11}$ reflects the fact that the test has a low specificity, since many repelling LCS cross the sampling point without implying any punctuated changes.

TABLE VI. Contingency table for hypothesis $\mathrm{H}_{11}$ for $12 \mathrm{~h}$ time scale.

\begin{tabular}{lcc}
\hline \hline & \multicolumn{2}{c}{ Punctuated change has occurred $\left(\Delta C_{p}\right)$} \\
\cline { 2 - 3 } Repelling LCS passed over & Yes & No \\
Kentland farm (L) & 7 & 6 \\
\hline Yes & 5 & 41 \\
No & & \multirow{2}{*}{} \\
\hline
\end{tabular}


TABLE VII. Contingency table for hypothesis $\mathrm{H}_{12}$ for $12 \mathrm{~h}$ time scale.

\begin{tabular}{lcc}
\hline \hline & \multicolumn{2}{c}{ Punctuated change has occurred $\left(\Delta C_{p}\right)$} \\
\cline { 2 - 3 } Attracting LCS passed over & Yes & No \\
Kentland farm (L) & 5 & 12 \\
\hline Yes & 7 & 35 \\
No & & \\
\hline \hline
\end{tabular}

The contingency table for testing hypothesis $\mathrm{H}_{12}$ is shown in Table IV.

The $p$-value for the data in Table IV corresponding to the hypothesis $\mathrm{H}_{12}$ on a $24 \mathrm{~h}$ time scale is 0.3313 . The sensitivity of the test for $\mathrm{H}_{12}$ is 0.3750 and the specificity is 0.7193 . The low sensitivity means that punctuated changes occur without the passage of attracting LCS over the sampling point, but the high specificity means that whenever an attracting LCS does pass the sampling point, the chance of a punctuated change occurring is high. The two-way correlation between the attracting LCS and punctuated changes using Eq. (10)is $\phi_{12}=0.085$.

For the combined hypothesis $\mathrm{H}_{1}$, the contingency table is given in Table $\mathrm{V}$.

The $p$-value for the data in Table $\mathrm{V}$ corresponding to the hypothesis $\mathrm{H}_{1}$ on a $24 \mathrm{~h}$ time scale is 0.0039 . The correlation for hypothesis $\mathrm{H}_{1}$ is $\phi=0.3384$ with a sensitivity of 0.8125 and specificity of 0.5695 . An inspection of the data shows that the low correlation and specificity is because of the high value of $n_{4}$, i.e., there are many repelling and attracting LCS which pass the sampling region without being associated with punctuated changes, but conversely, most punctuated changes are due to the passage of either a repelling or an attracting LCS. The high $p$-value $(0.3313)$ for hypothesis $\mathrm{H}_{12}$ means that we cannot reject the null hypothesis for this case, despite the high value of specificity. When we consider the hypotheses for the repelling LCS $\left(\mathrm{H}_{11}\right)$ and combined LCS $\left(\mathrm{H}_{1}\right)$, the high sensitivity and low $p$-value allow us to reject the null hypothesis $\mathrm{H}_{0}$ for the 24-h time scale. We repeat the same calculations for the $12 \mathrm{~h}$ time scale below.

For the data in Table IV corresponding to the hypothesis $\mathrm{H}_{11}$ on a $12 \mathrm{~h}$ time scale, $p=0.0022$, the sensitivity is $s_{2}=0.5833$, and the specificity is $s_{1}=0.8723$. For the data in Table VII corresponding to the hypothesis $\mathrm{H}_{12}$ on a $12 \mathrm{~h}$ time scale, $p=0.2245, s_{2}=0.7447$, and $s_{1}=0.4167$. For the data in Table VIII corresponding to the hypothesis $\mathrm{H}_{1}$ on a $12 \mathrm{~h}$ time scale, $p=0.0027, s_{2}=0.8333$, and $s_{1}=0.6596$. The high $p$-value $(0.2245)$ for hypothesis $\mathrm{H}_{12}$ means that we cannot reject the null hypothesis for this case, despite the high value of specificity. When we consider the hypotheses for the forward LCS and combined LCS, the high sensitivity and low $p$-value allow us to reject the null hypothesis $\mathrm{H}_{0}$ for the $12 \mathrm{~h}$ time scale as well.

\section{CONCLUSIONS AND FUTURE WORK}

We have outlined a geometric framework of ATBs, which the evidence suggests are associated with punctuated changes in atmospheric concentrations of viable microorganisms of the genus Fusarium, collected with autonomous UAVs. Our autonomous UAV sampling scheme (consistent airspeed, sam-
TABLE VIII. Contingency table for hypothesis $\mathrm{H}_{1}$ for $12 \mathrm{~h}$ time scale.

\begin{tabular}{lcc}
\hline \hline & \multicolumn{2}{c}{ Punctuated change has occurred $\left(\Delta C_{p}\right)$} \\
\cline { 2 - 3 } Attracting or repelling LCS passed & Yes & No \\
\cline { 2 - 3 } over Kentland farm (L) & 10 & 16 \\
Yes & 2 & 31 \\
No & & \\
\hline \hline
\end{tabular}

pling pattern, and altitude throughout the sampling missions) provides us with a robust measure of the aerial concentration of Fusarium, suggesting that any variation in the concentration is attributable to factors other than the accuracy of the sampling regime (e.g., an association with atmospheric structures). ATBs were identified with attracting and repelling LCSs obtained from trajectory computations using meteorological data in an equation-free manner. An analysis of the FTLE field for periods surrounding the collection of Fusarium with UAVs showed that punctuated changes in the concentration of Fusarium were associated with the movement of repelling LCS. While the concept of transport barriers have been applied in earlier works to study transport in the upper atmosphere ${ }^{15}$ and lobe dynamics in hurricanes, ${ }^{17}$ to our knowledge this is the first detailed application of the application of LCS to study meso- to synoptic-scale transport and punctuated changes of concentration of a microorganisms in the lower atmosphere. This work also sets a framework for the study of transport of arbitrary tracers in the oceans and the atmosphere, in particular, the punctuated changes of tracer concentration.

To summarize the results of the hypothesis testing, our analysis suggests that punctuated changes in the atmospheric concentrations of Fusarium are associated with the movement of LCSs, and particularly repelling LCSs, in a one-way correlation. That is, when a punctuated change occurs between two sampling times, there is a high probability that a repelling LCS passed over the sampling location between the two sampling times. We are not suggesting a two-way correlation, i.e., the movement of every repelling LCS does not lead to a punctuated change. There are two potential explanations for this. First, the chaotic nature of atmospheric flow ensures that many trajectories have a high local repulsion. Second, we did not discriminate between ridges in the FTLE field of high and low magnitude above the threshold. It is possible that the magnitude of punctuated changes can depend on "repelling strength" of the repelling LCS, e.g., the repulsion ratio defined by Haller. ${ }^{39}$ Furthermore, the results of our hypotheses testing and the $p$ values are influenced by several parameters: the time-scale used to determine punctuated changes, (12 or $24 \mathrm{~h}$ ), the baseline concentration of spores, the threshold value of $\Delta C$ which determines whether a change is significant, and the threshold value for the FTLE field, $\sigma_{\text {min }}$. Future studies are expected to systematically investigate the influence of these factors.

Further analyses of the cultures of Fusarium collected during the sampling missions analyzed as part of this work will help identify the population structure of the Fusarium in each of the samples. This will allow us to consider not just total concentration of spores in the Fusarium genus, as we considered here, but to resolve down to the level of individual 
species. Each species becomes an independent bio-tracer in the atmosphere and will allow more rigorous testing of the ATB hypotheses. Moreover, future studies with UAVs at multiple, well-spaced, geographic locations may help resolve the spatial variations in the punctuated changes. This will also help to test if the "strength" of an LCS has any correlation with the magnitude of the punctuated changes.

For the present study, we did not consider the reasons why punctuated changes in Fusarium would be associated with ATBs. Further investigation of the conditions which give rise to this association will be forthcoming. It is hoped that the ATB hypothesis will eventually translate into predictive power, finding useful applications in forecasting the movement of invasive airborne microbes. Ideally this method could contribute to existing information systems for pest management and disease control, such as the integrated pest/management and pest information platform for extension and education (IPM PIPE) $)^{57,58}$ and the North American Plant Disease Forecasting Center. ${ }^{59}$ Finally, while we have focused only on the punctuated changes in the concentration of Fusarium, our hypotheses are more generally applicable and could be applied to other microorganisms and chemical pollutants in the atmospheres and oceans.

\section{ACKNOWLEDGMENTS}

We thank Benjamin Dingus and John Cianchetti for their tireless dedication in the development and operation of the autonomous UAVs used to collect the colonies of Fusarium reported in this work. We wish to thank the following colleagues for their helpful discussions and comments: Don Aylor, Ken Bowman, Amir BozorgMagham, Philip Du Toit, George Haller, Cheryl Harrison, Scott Isard, Scott Kelly, Francois Lekien, Jerrold Marsden, Joseph Russo, Carmine Senatore, Mark Stremler, and Craig Woolsey. This material is based upon work supported by the National Science Foundation under Grant No. 0919088. Any opinions, findings, and conclusions or recommendations expressed in this material are those of the authors and do not necessarily reflect the views of the National Science Foundation.

\section{APPENDIX: SPORE CONCENTRATION DATA}

This appendix provides, in Tables IX and $\mathrm{X}$, the data on concentration of atmospheric Fusarium spores (in spores $/ \mathrm{m}^{3}$ ), the date and time at which the sample was collected, and the categorical variables as described in Sec. III C.

TABLE IX. Data on concentration of Fusarium in samples collected with UAVs 100m above ground level at Virginia Tech's Kentland Farm and passage of LCSs.

\begin{tabular}{|c|c|c|c|c|c|c|c|}
\hline Date & Time (UTC) & $\mathrm{C}$ & $\Delta \mathrm{C}$ & $\Delta \mathrm{C} / \mathrm{C}$ & Punctuated change & Repelling LCS & Attracting LCS \\
\hline $9 / 16 / 2006$ & $17: 15$ & 16.19906 & - & - & & & \\
\hline $9 / 16 / 2006$ & 19:00 & 14.81614 & -1.38292 & -0.085370644 & $\mathrm{~N}$ & $\mathrm{~N}$ & $\mathrm{Y}$ \\
\hline $9 / 16 / 2006$ & $20: 30$ & 8.783851 & -6.03229 & -0.407143011 & $\mathrm{Y}$ & $\mathrm{N}$ & $\mathrm{Y}$ \\
\hline $9 / 29 / 2006$ & $22: 35$ & 6.145202 & - & - & & & \\
\hline $9 / 30 / 2006$ & $15: 10$ & 1.30206 & 4.84314 & -0.788117632 & $\mathrm{Y}$ & $\mathrm{Y}$ & $\mathrm{N}$ \\
\hline $9 / 30 / 2006$ & $15: 50$ & 9.639299 & 8.337239 & 6.403114837 & $\mathrm{Y}$ & $\mathrm{N}$ & $\mathrm{N}$ \\
\hline $10 / 8 / 2006$ & $14: 45$ & 0.484678 & - & - & & & \\
\hline $10 / 8 / 2006$ & $15: 30$ & 3.734943 & 3.250265 & 6.706030724 & $\mathrm{Y}$ & $\mathrm{N}$ & $\mathrm{Y}$ \\
\hline $10 / 9 / 2006$ & $20: 35$ & 3.014947 & -0.72 & -0.192773077 & $\mathrm{Y}$ & $\mathrm{Y}$ & $\mathrm{Y}$ \\
\hline $10 / 9 / 2006$ & $21: 15$ & 1.65812 & -1.35683 & -0.450033322 & $\mathrm{~N}$ & $\mathrm{~N}$ & $\mathrm{~N}$ \\
\hline $10 / 9 / 2006$ & $22: 00$ & 3.377004 & 1.718884 & 1.036646101 & $\mathrm{~N}$ & $\mathrm{~N}$ & $\mathrm{~N}$ \\
\hline $10 / 21 / 2006$ & $12: 00$ & 1.09637 & - & - & & & \\
\hline $10 / 21 / 2006$ & $19: 15$ & 1.3284 & 0.23203 & 0.211634615 & $\mathrm{~N}$ & $\mathrm{~N}$ & $\mathrm{~N}$ \\
\hline $11 / 10 / 2006$ & $15: 50$ & 1.814305 & - & - & $\mathrm{N}$ & $\mathrm{Y}$ & $\mathrm{N}$ \\
\hline $11 / 10 / 2006$ & $16: 25$ & 0.329732 & -1.48457 & -0.818259854 & $\mathrm{~N}$ & $\mathrm{~N}$ & $\mathrm{~N}$ \\
\hline $11 / 10 / 2006$ & $21: 25$ & 1.805832 & 1.4761 & 4.476664004 & $\mathrm{~N}$ & $\mathrm{~N}$ & $\mathrm{Y}$ \\
\hline $11 / 10 / 2006$ & $21: 55$ & 4.099233 & 2.293401 & 1.269997166 & $\mathrm{Y}$ & $\mathrm{N}$ & $\mathrm{Y}$ \\
\hline $11 / 11 / 2006$ & $17: 05$ & 1.719432 & -2.3798 & -0.580547826 & $\mathrm{Y}$ & $\mathrm{Y}$ & $\mathrm{Y}$ \\
\hline $11 / 11 / 2006$ & $18: 20$ & 2.742224 & 1.022792 & 0.594842838 & $\mathrm{~N}$ & $\mathrm{~N}$ & $\mathrm{~N}$ \\
\hline $11 / 24 / 2006$ & $20: 40$ & 0.342483 & - & - & & & \\
\hline $11 / 25 / 2006$ & $16: 45$ & 0.340983 & -0.0015 & -0.004379366 & $\mathrm{~N}$ & $\mathrm{Y}$ & $\mathrm{Y}$ \\
\hline $11 / 25 / 2006$ & $17: 15$ & 0.342708 & 0.001725 & 0.005060047 & $\mathrm{~N}$ & $\mathrm{~N}$ & $\mathrm{Y}$ \\
\hline $11 / 25 / 2006$ & $17: 45$ & 0 & -0.34271 & -1 & $\mathrm{~N}$ & $\mathrm{~N}$ & $\mathrm{~N}$ \\
\hline $11 / 25 / 2006$ & $18: 30$ & 0 & 0 & - & $\mathrm{N}$ & $\mathrm{Y}$ & $\mathrm{N}$ \\
\hline $11 / 25 / 2006$ & 20:00 & 0 & 0 & - & $\mathrm{N}$ & $\mathrm{N}$ & $\mathrm{N}$ \\
\hline $11 / 25 / 2006$ & $20: 30$ & 0.341449 & 0.341449 & - & $\mathrm{N}$ & $\mathrm{N}$ & $\mathrm{N}$ \\
\hline $4 / 30 / 2007$ & $14: 15$ & 0.864954 & - & - & & & \\
\hline $4 / 30 / 2007$ & $15: 00$ & 0.427646 & -0.43731 & -0.505585562 & $\mathrm{~N}$ & $\mathrm{Y}$ & $\mathrm{N}$ \\
\hline $5 / 1 / 2007$ & $14: 00$ & 12.32774 & 11.9001 & 27.82699456 & $\mathrm{Y}$ & $\mathrm{Y}$ & $\mathrm{Y}$ \\
\hline $5 / 1 / 2007$ & $15: 00$ & 7.206588 & -5.12116 & -0.415417151 & $\mathrm{Y}$ & $\mathrm{Y}$ & $\mathrm{N}$ \\
\hline $5 / 1 / 2007$ & $20: 15$ & 2.151275 & -5.05531 & -0.70148494 & $\mathrm{Y}$ & $\mathrm{Y}$ & $\mathrm{Y}$ \\
\hline $5 / 1 / 2007$ & $22: 00$ & 1.231439 & -0.91984 & -0.427577368 & $\mathrm{~N}$ & $\mathrm{~N}$ & $\mathrm{~N}$ \\
\hline $5 / 2 / 2007$ & $14: 00$ & 2.152531 & 0.921092 & 0.747980822 & $\mathrm{~N}$ & $\mathrm{Y}$ & $\mathrm{Y}$ \\
\hline
\end{tabular}


TABLE X. Data on concentration of Fusarium in samples and passage of LCSs.

\begin{tabular}{|c|c|c|c|c|c|c|c|}
\hline Date & Time (UTC) & $\mathrm{C}$ & $\Delta \mathrm{C}$ & $\Delta \mathrm{C} / \mathrm{C}$ & Punctuated change & Repelling LCS & Attracting LCS \\
\hline $5 / 8 / 2007$ & 18:00 & 2.065564 & - & - & & & \\
\hline $5 / 8 / 2007$ & $22: 00$ & 0.855901 & -1.20966 & -0.585633155 & $\mathrm{~N}$ & $\mathrm{Y}$ & $\mathrm{Y}$ \\
\hline $5 / 9 / 2007$ & $14: 00$ & 0.42531 & -0.43059 & -0.503085782 & $\mathrm{~N}$ & $\mathrm{Y}$ & $\mathrm{Y}$ \\
\hline $5 / 9 / 2007$ & $15: 00$ & 0 & -0.42531 & -1 & $\mathrm{~N}$ & $\mathrm{~N}$ & $\mathrm{~N}$ \\
\hline $5 / 9 / 2007$ & $16: 00$ & 0.867592 & 0.867592 & - & $\mathrm{N}$ & $\mathrm{N}$ & $\mathrm{N}$ \\
\hline $5 / 9 / 2007$ & $17: 00$ & 1.709266 & 0.841674 & 0.970127005 & $\mathrm{~N}$ & $\mathrm{~N}$ & $\mathrm{~N}$ \\
\hline $5 / 9 / 2007$ & $18: 00$ & 0.861002 & -0.84826 & -0.496273798 & $\mathrm{~N}$ & $\mathrm{~N}$ & $\mathrm{~N}$ \\
\hline $5 / 9 / 2007$ & 20:00 & 4.284671 & 3.423669 & 3.97637636 & $\mathrm{Y}$ & $\mathrm{N}$ & $\mathrm{N}$ \\
\hline $5 / 9 / 2007$ & $21: 00$ & 4.756824 & 0.472153 & 0.110195852 & $\mathrm{~N}$ & $\mathrm{~N}$ & $\mathrm{~N}$ \\
\hline $5 / 9 / 2007$ & $22: 00$ & 1.723555 & -3.03327 & -0.63766688 & $\mathrm{Y}$ & $\mathrm{Y}$ & $\mathrm{N}$ \\
\hline $5 / 10 / 2007$ & $14: 00$ & 2.156161 & 0.432606 & 0.250996381 & $\mathrm{~N}$ & $\mathrm{Y}$ & $\mathrm{N}$ \\
\hline $5 / 10 / 2007$ & $15: 00$ & 3.880779 & 1.724618 & 0.799855845 & $\mathrm{Y}$ & $\mathrm{Y}$ & $\mathrm{N}$ \\
\hline $5 / 10 / 2007$ & $16: 00$ & 1.26961 & -2.61117 & -0.672846739 & $\mathrm{Y}$ & $\mathrm{Y}$ & $\mathrm{N}$ \\
\hline $5 / 11 / 2007$ & $18: 00$ & 0.853422 & -0.41619 & -0.327807705 & $\mathrm{~N}$ & $\mathrm{Y}$ & $\mathrm{N}$ \\
\hline $5 / 11 / 2007$ & 19:00 & 0.8483 & -0.00512 & -0.006001623 & $\mathrm{~N}$ & $\mathrm{~N}$ & $\mathrm{Y}$ \\
\hline $5 / 11 / 2007$ & $20: 00$ & 1.276074 & 0.427774 & 0.504272155 & $\mathrm{~N}$ & $\mathrm{~N}$ & $\mathrm{~N}$ \\
\hline $5 / 11 / 2007$ & $21: 00$ & 1.283445 & 0.007371 & 0.00577659 & $\mathrm{~N}$ & $\mathrm{~N}$ & $\mathrm{~N}$ \\
\hline $5 / 14 / 2007$ & $17: 15$ & 0.855144 & - & - & & & \\
\hline $5 / 14 / 2007$ & 18:00 & 2.571325 & 1.71618 & 2.006889493 & $\mathrm{~N}$ & $\mathrm{~N}$ & $\mathrm{~N}$ \\
\hline $5 / 14 / 2007$ & 19:00 & 2.9695 & 0.398175 & 0.154852139 & $\mathrm{~N}$ & $\mathrm{~N}$ & $\mathrm{~N}$ \\
\hline $5 / 17 / 2007$ & $15: 15$ & 0 & - & - & & & \\
\hline $5 / 17 / 2007$ & $16: 00$ & 0 & 0 & - & $\mathrm{N}$ & $\mathrm{N}$ & $\mathrm{N}$ \\
\hline $5 / 24 / 2007$ & 00:30 & 1.284502 & - & - & & & \\
\hline $5 / 24 / 2007$ & 01:30 & 0.501114 & -0.78339 & -0.609876436 & $\mathrm{~N}$ & $\mathrm{~N}$ & $\mathrm{~N}$ \\
\hline $5 / 24 / 2007$ & $02: 30$ & 1.526163 & 1.025048 & 2.045537218 & $\mathrm{~N}$ & $\mathrm{~N}$ & $\mathrm{~N}$ \\
\hline $10 / 19 / 2009$ & $19: 48$ & 1.549173 & - & - & $\mathrm{N}$ & & \\
\hline $10 / 19 / 2009$ & $20: 40$ & 0.860652 & -0.68852 & -0.444444444 & $\mathrm{~N}$ & $\mathrm{~N}$ & $\mathrm{~N}$ \\
\hline $11 / 09 / 2009$ & $15: 30$ & 1.290978 & - & - & & & \\
\hline $11 / 09 / 2009$ & $17: 35$ & 0.21936 & -1.07162 & -0.830081884 & $\mathrm{~N}$ & $\mathrm{~N}$ & $\mathrm{~N}$ \\
\hline $11 / 13 / 2009$ & $14: 46$ & 0.545154 & 0 & 0 & & & \\
\hline $11 / 13 / 2009$ & $15: 57$ & 0.327092 & -0.21806 & -0.4 & $\mathrm{~N}$ & $\mathrm{~N}$ & $\mathrm{Y}$ \\
\hline $11 / 13 / 2009$ & $17: 46$ & 0.817731 & 0.490638 & 1.5 & $\mathrm{~N}$ & $\mathrm{~N}$ & $\mathrm{Y}$ \\
\hline $11 / 13 / 2009$ & 19:09 & 1.144823 & 0.327092 & 0.4 & $\mathrm{~N}$ & $\mathrm{~N}$ & $\mathrm{Y}$ \\
\hline $11 / 16 / 2009$ & $14: 41$ & 0 & - & - & & & \\
\hline $11 / 16 / 2009$ & $15: 45$ & 0.109031 & 0.109031 & - & $\mathrm{N}$ & $\mathrm{N}$ & $\mathrm{Y}$ \\
\hline $11 / 16 / 2009$ & $18: 23$ & 0.654184 & 0.545154 & 4.999999998 & $\mathrm{~N}$ & $\mathrm{~N}$ & $\mathrm{~N}$ \\
\hline $11 / 16 / 2009$ & $19: 45$ & 3.598014 & 2.94383 & 4.500000001 & $\mathrm{Y}$ & $\mathrm{Y}$ & $\mathrm{N}$ \\
\hline $11 / 17 / 2009$ & $15: 50$ & 0.436123 & -3.16189 & -0.878787879 & $\mathrm{Y}$ & $\mathrm{Y}$ & $\mathrm{Y}$ \\
\hline $11 / 17 / 2009$ & $16: 45$ & 0.109031 & -0.32709 & -0.75 & $\mathrm{~N}$ & $\mathrm{~N}$ & $\mathrm{~N}$ \\
\hline $11 / 17 / 2009$ & $17: 45$ & 0.109031 & 0 & 0 & $\mathrm{~N}$ & $\mathrm{~N}$ & $\mathrm{~N}$ \\
\hline $01 / 14 / 2010$ & $18: 30$ & 0.327092 & - & - & & & \\
\hline $01 / 14 / 2010$ & $19: 45$ & 0.327092 & 0 & 0 & $\mathrm{~N}$ & $\mathrm{~N}$ & $\mathrm{~N}$ \\
\hline $01 / 15 / 2010$ & $15: 35$ & 0.218061 & -0.10903 & -0.333333333 & $\mathrm{~N}$ & $\mathrm{Y}$ & $\mathrm{N}$ \\
\hline $01 / 15 / 2010$ & $16: 40$ & 0.436123 & 0.218061 & 1 & $\mathrm{~N}$ & $\mathrm{~N}$ & $\mathrm{~N}$ \\
\hline 03/09/2010 & $16: 11$ & 1.090307 & - & - & & & \\
\hline 03/09/2010 & $17: 53$ & 0.332713 & -0.75759 & -0.694844776 & $\mathrm{~N}$ & $\mathrm{Y}$ & $\mathrm{Y}$ \\
\hline $03 / 09 / 2010$ & 19:06 & 0.816995 & 0.484282 & 1.455555555 & $\mathrm{~N}$ & $\mathrm{Y}$ & $\mathrm{Y}$ \\
\hline $03 / 10 / 2010$ & $14: 29$ & 0.24985 & -0.56715 & -0.694184414 & $\mathrm{~N}$ & $\mathrm{Y}$ & $\mathrm{N}$ \\
\hline 03/10/2010 & $15: 35$ & 1.204633 & 0.954783 & 3.821428572 & $\mathrm{~N}$ & $\mathrm{~N}$ & $\mathrm{~N}$ \\
\hline $03 / 10 / 2010$ & $16: 30$ & 0.687087 & -0.51755 & -0.42962963 & $\mathrm{~N}$ & $\mathrm{~N}$ & $\mathrm{~N}$ \\
\hline $03 / 10 / 2010$ & $18: 00$ & 0.437237 & -0.24985 & -0.363636364 & $\mathrm{~N}$ & $\mathrm{~N}$ & $\mathrm{~N}$ \\
\hline 03/10/2010 & 19:00 & 0.812012 & 0.374775 & 0.857142857 & $\mathrm{~N}$ & $\mathrm{~N}$ & $\mathrm{~N}$ \\
\hline
\end{tabular}

${ }^{1}$ R. A. Duce, C. K. Unni, B. J. Ray, J. M. Prospero, and J. T. Merrill, Science 209, 1522 (1980).

${ }^{2}$ J. R. Parrington, W. H. Zoller, and N. K. Aras, Science 220, 195 (1983).

${ }^{3}$ D. E. Aylor, Agric. Forest Meteorol. 38, 263 (2002).

${ }^{4}$ S. J. Piketh, R. J. Swap, W. Maenhaut, H. J. Annergarn, and P. Formenti, J. Geophys. Res. 107, 4817 (2002).

${ }^{5}$ E. A. Shinn, D. W. Griffin, and D. B. Seba, Arch. Environ. Health 58 (8), 498 (2003).
${ }^{6}$ C. A. Rogers and E. Levetin, Int. J. Biometeorol. 42, 65 (1998).

${ }^{7}$ S. Isard and S. H. Gage, Flow of Life in the Atmosphere: An Airscape Approach to Understanding Invasive Organisms (Michigan State University, East Lansing, MI, 2001).

${ }^{8}$ R. T. Pierrehumbert, Geophys. Astrophys. Fluid Dyn. 58, 285 (1991).

${ }^{9}$ R. T. Pierrehumbert, Phys. Fluids 3A, 1250 (1991).

${ }^{10}$ S. Wiggins, Annu. Rev. Fluid Mech. 37, 295 (2005). 
${ }^{11}$ K. Ide, D. Small, and S. Wiggins, Nonlinear Processes Geophys. 9, 237 (2002).

${ }^{12}$ C. Coulliette, F. Lekien, J. D. Paduan, G. Haller, and J. Marsden, Environ. Sci. Technol. 41, 6562 (2007).

${ }^{13}$ K. P. Bowman, L. L. Pan, T. Campos, and R. Gao, J. Geophys. Res. 112, D18111 (2007).

${ }^{14}$ L. L. Pan, K. P. Bowman, E. Atlas, S. C. Wofsy, F. Zhang, J. F. Bresch, B. A. Ridley, J. V. Pittman, C. Homeyer, P. Romashkin, and W. A. Cooper, Bull. Am. Meteorol. Soc. 91, D23102 (2010).

${ }^{15}$ F. Lekien and S. D. Ross, Chaos 20, 017505 (2010).

${ }^{16}$ W. Tang, M. Mathur, G. Haller, D. C. Hahn, and F. H. Ruggiero, J. Atmos. Sci. 67, 2307 (2010).

${ }^{17}$ P. C. Du Toit and J. E. Marsden, J. Fixed Point Theory Appl. 7 (2), 351 (2010).

${ }^{18}$ M. P. McMullen, R. Jones, and D. Gallenberg, Plant Dis. 81, 1340 (1997).

${ }^{19}$ M. Ichinoe, H. Kurata, Y. Sugiura, and Y. Ueno, Appl. Environ. Microbiol. 46, 1364 (1983).

${ }^{20}$ B. J. Bush, M. L. Carson, M. A. Cubeta, W. M. Hagler, and G. A. Payne, Phytopathology 46, 88 (2004).

${ }^{21} \mathrm{~W}$. Zdunkowski and A. Bott, Dynamics of the Atmosphere: A Course in Theoretical Meteorology (Cambridge University Press, Cambridge, New York, 2003).

${ }^{22}$ J. R. Holton, An Introduction to Dynamic Meteorology, 4th ed. (Elsevier Academic Press, Burlington, MA, 2004).

${ }^{23}$ M. T. Boehm and D. E. Aylor, Atmos. Environ. 39, 4841 (2005).

${ }^{24} \mathrm{~A}$. Majda, Introduction to PDEs and Waves for the Atmosphere and Ocean (American Mathematical Society, Providence, RI, 2003).

${ }^{25}$ D. E. Aylor and T. K. Flesch, J. Appl. Meterorol. 40, 1196 (2001).

${ }^{26}$ D. E. Aylor, J. Aerosol Sci. 33, 1599 (2003).

${ }^{27}$ D. G. Schmale and G. C. Bergstrom, Can. J. Plant Pathol. 26, 591 (2004).

${ }^{28}$ D. G. Schmale, E. J. Shields, and G. C. Bergstrom, Can. J. Plant Pathol. 28, 1 (2006).

${ }^{29}$ D. E. Aylor, Ecology 84, 1989 (2003).

${ }^{30}$ J. M. Davis, Annu. Rev. Phytopathol. 25, 169 (1987).

${ }^{31}$ D. W. Griffin, Clin. Microbiol. Rev. 20(3), 459 (2007).

${ }^{32}$ S. C. Shadden, F. Lekien, and J. Marsden, Physica D 212, 271 (2005).

${ }^{33}$ F. Lekien, S. C. Shadden, and J. E. Marsden, J. Math. Phys. 48, 065404 (2007).

${ }^{34}$ P. Tallapragada and S. D. Ross, Phys. Rev. E 78 (2008).

${ }^{35}$ E. S. Gawlik, J. E. Marsden, P. C. Du Toit, and S. Campagnola, Celest. Mech. Dyn. Astron. 3, 227 (2009).

${ }^{36}$ C. Senatore and S. D. Ross, Int. J. Numer. Methods Eng. 86, 1163 (2011).
${ }^{37}$ W. Tang, G. Haller, and P. W. Chan, J. Appl. Meteorol. Clim. 50, 325 (2011).

${ }^{38} \mathrm{~B}$. Cushman-Roisin and J. M. Beckers, Introduction to Geophysical Fluid Dynamics (Academic, New York, 2011).

${ }^{39}$ G. Haller, Phys. Fluids A13, 3368 (2001).

${ }^{40}$ G. Haller, Physica D 240, 574 (2011).

${ }^{41}$ F. J. Beron-Vera, M. J. Olascoaga, M. G. Brown, H. Kocak, and I. Rypina, Chaos 20, 017514 (2010).

${ }^{42}$ J. Peng and J. O. Dabiri, J. Fluid Mech. 623, 75 (2009).

${ }^{43}$ M. Wilson, J. Peng, J. O. Dabiri, and J. D. Eldredge, J. Phys.: Condens. Matter 21, 204105 (2009).

${ }^{44}$ S. L. Maldonado-Ramirez, D. G. Schmale, E. J. Shields, and G. C. Bergstrom, Agric. Forest Meteorol. 132, 20 (2005).

${ }^{45}$ D. G. Schmale, B. R. Dingus, and C. Reinholtz, J. Field Robot. 25, 133 (2008).

${ }^{46}$ L. Techy, D. G. Schmale, and C. A. Woolsey, J. Field Robot. 27, 335 (2010).

${ }^{47}$ D. G. Schmale, J. F. Leslie, K. A. Zeller, A. A. Saleh, E. J. Shields, and G. C. Bergstrom, Phytopathology 96, 1021 (2006).

${ }^{48}$ D. E. Aylor, M. T. Boehm, and E. J. Shields, J. Appl. Meteorol. Clim. 45, 1003 (2006)

${ }^{49}$ D. G. Schmale, Q. A. Arntsen, and G. C. Bergstrom, Can. J. Plant Pathol. 27, 376 (2005).

${ }^{50}$ A. J. Prussin II, M. A. Stremler, and D. G. Schmale III, A fluid dynamics approach to determine the aerobiological sampling efficiency of autonomous unmanned aerial vehicles (UAVs) (2011). NSF-IGERT Poster Competition.

${ }^{51}$ D. G. Schmale, S. D. Ross, T. L. Fetters, P. Tallapragada, A. K. WoodJones, and B. Dingus, Isolates of Fusarium graminearum collected 40-320 meters above ground level cause Fusarium head blight in wheat and produce trichothecene mycotoxins. Aerobiologia (2011).

${ }^{52}$ B. Joseph and B. Legras, J. Atmos. Sci. 59, 1198 (2002).

${ }^{53}$ T. Koh and B. Legras, Chaos 12 (2), 382 (2002).

${ }^{54}$ A. Agresti. Categorical Data Analysis, 1st ed. (John Wiley and Sons Inc., Hoboken, NJ, 1990).

${ }^{55}$ G. K. Rutledge, J. Alpert, and W. Ebuisaki, Bull. Amer. Meteor. Soc. 87, 327 (2006).

${ }^{56}$ F. Lekien and J. Marsden, Int. J. Numer. Methods Eng. 63, 455 (2005).

${ }^{57}$ P. C. Du Toit, "Transport and separatrices in time dependent flows," Ph.D. thesis, California Institute of Technology, 2010.

${ }^{58}$ S. A. Isard, J. M. Russo, and E. D. DeWolf, Plant Health Progress (2006).

${ }^{59}$ C. E. Main, Blue Mold Historical Forecasts (North American Plant Disease Forecast Center); Archives for Blue mold (unpublished). 